\title{
NORMAL FAMILIES OF MEROMORPHIC MAPPINGS OF SEVERAL COMPLEX VARIABLES FOR MOVING HYPERSURFACES IN A COMPLEX PROJECTIVE SPACE
}

\author{
GERD DETHLOFF, DO DUC THAI, AND \\ PHAM NGUYEN THU TRANG
}

\begin{abstract}
The main aim of this article is to give sufficient conditions for a family of meromorphic mappings of a domain $D$ in $\mathbb{C}^{n}$ into $\mathbb{P}^{N}(\mathbb{C})$ to be meromorphically normal if they satisfy only some very weak conditions with respect to moving hypersurfaces in $\mathbb{P}^{N}(\mathbb{C})$, namely, that their intersections with these moving hypersurfaces, which moreover may depend on the meromorphic maps, are in some sense uniform. Our results generalize and complete previous results in this area, especially the works of Fujimoto, Tu, Tu-Li, Mai-ThaiTrang, and the recent work of Quang-Tan.
\end{abstract}

\section{$\S 1$. Introduction}

Classically, a family $\mathcal{F}$ of holomorphic functions on a domain $D \subset \mathbb{C}$ is said to be (holomorphically) normal if every sequence in $\mathcal{F}$ contains a subsequence which converges uniformly on every compact subset of $D$ to a holomorphic map from $D$ into $P^{1}$.

In 1957, Lehto and Virtanen [7] introduced the concept of normal meromorphic functions in connection with the study of boundary behavior of meromorphic functions of one complex variable. Since then normal families of holomorphic maps have been studied intensively, resulting in an extensive development in the one-complex-variable context and in generalizations to the several-complex-variables setting (see [1], [5], [6], [23], and references cited in [5] and [23]).

The first ideas and results on normal families of meromorphic mappings of several complex variables were introduced by Rutishauser [14] and Stoll [15].

Received March 20, 2013. Revised January 21, 2014. Accepted February 5, 2014.

First published online January 27, 2015.

2010 Mathematics Subject Classification. Primary 32A19; Secondary 32H30. 
The notion of a meromorphically normal family into the $N$-dimensional complex projective space was introduced by Fujimoto [4]. (See Section 2.5 below for the definition of these concepts.) Fujimoto in [4] also gave some sufficient conditions for a family of meromorphic mappings of a domain $D$ in $\mathbb{C}^{n}$ into $\mathbb{P}^{N}(\mathbb{C})$ to be meromorphically normal. In 2002, Tu [21] considered meromorphically normal families of meromorphic mappings of a domain $D$ in $\mathbb{C}^{n}$ into $\mathbb{P}^{N}(\mathbb{C})$ for hyperplanes. Generalizing the above results of Fujimoto and Tu, in 2005 Mai, Thai, and Trang [8] gave a sufficient condition for the meromorphic normality of a family of meromorphic mappings of a domain $D$ in $\mathbb{C}^{n}$ into $\mathbb{P}^{N}(\mathbb{C})$ for fixed hypersurfaces, as follows. (See Section 2 below for the necessary definitions, in particular Section 2.3 for the definition of $D(\cdots)$.)

Theorem A ([8, Theorem A]). Let $\mathcal{F}$ be a family of meromorphic mappings of a domain $D$ in $\mathbb{C}^{n}$ into $\mathbb{P}^{N}(\mathbb{C})$. Suppose that for each $f \in \mathcal{F}$ there exist $q \geq 2 N+1$ hypersurfaces $H_{1}(f), H_{2}(f), \ldots, H_{q}(f)$ in $\mathbb{P}^{N}(\mathbb{C})$ with

$$
\inf \left\{D\left(H_{1}(f), \ldots, H_{q}(f)\right), f \in \mathcal{F}\right\}>0
$$

and

$$
f(D) \not \subset H_{i}(f) \quad(1 \leq i \leq N+1),
$$

where $q$ is independent of $f$, but the hypersurfaces $H_{i}(f)$ may depend on $f$, such that the following two conditions are satisfied.

(i) For any fixed compact subset $K$ of $D$, the $2(n-1)$-dimensional Lebesgue areas of $f^{-1}\left(H_{i}(f)\right) \cap K(1 \leq i \leq N+1)$ with counting multiplicities are bounded above for all $f$ in $\mathcal{F}$.

(ii) There exists a closed subset $S$ of $D$ with $\Lambda^{2 n-1}(S)=0$ such that, for any fixed compact subset $K$ of $D-S$, the $2(n-1)$-dimensional Lebesgue areas of $f^{-1}\left(H_{i}(f)\right) \cap K(N+2 \leq i \leq q)$ with counting multiplicities are bounded above for all $f$ in $\mathcal{F}$.

Then $\mathcal{F}$ is a meromorphically normal family on $D$.

Recently, motivated by the investigation of value distribution theory for moving hyperplanes (e.g., in [12], [13], [16]-[18]), the study of the normality of families of meromorphic mappings of a domain $D$ in $\mathbb{C}^{n}$ into $\mathbb{P}^{N}(\mathbb{C})$ for moving hyperplanes or hypersurfaces has started. While a substantial amount of information has been amassed concerning the normality of families of meromorphic mappings for fixed targets through the years, the present knowledge of this problem for moving targets has remained meagre. There 
are only a few such results in some restricted situations (see [11], [22]). For instance, we recall a recent result of Quang and Tan [11] which is the best result available at present and which generalizes [22, Theorem 2.2].

Theorem B ([11, Theorem 1.4]). Let $\mathcal{F}$ be a family of meromorphic mappings of a domain $D \subset \mathbb{C}^{n}$ into $\mathbb{P}^{N}(\mathbb{C})$, and let $Q_{1}, \ldots, Q_{q}(q \geq 2 N+1)$ be $q$ moving hypersurfaces in $\mathbb{P}^{N}(\mathbb{C})$ in (weakly) general position such that

(i) for any fixed compact subset $K$ of $D$, the $2(n-1)$-dimensional Lebesgue areas of $f^{-1}\left(Q_{j}\right) \cap K(1 \leq j \leq N+1)$ counting multiplicities are uniformly bounded above for all $f$ in $\mathcal{F}$; and

(ii) there exists a thin analytic subset $S$ of $D$ such that for any fixed compact subset $K$ of $D$, the $2(n-1)$-dimensional Lebesgue areas of $f^{-1}\left(Q_{j}\right) \cap$ $(K-S)(N+2 \leq j \leq q)$ regardless of multiplicities are uniformly bounded above for all $f$ in $\mathcal{F}$.

Then $\mathcal{F}$ is a meromorphically normal family on $D$.

We would like to emphasize that, in Theorem B, the $q$ moving hypersurfaces $Q_{1}, \ldots, Q_{q}$ in $\mathbb{P}^{N}(\mathbb{C})$ are independent on $f \in \mathcal{F}$ (i.e., are common for all $f \in \mathcal{F}$ ). Thus, the following question arose naturally at this point: Does Theorem A hold for moving hypersurfaces $H_{1}(f), H_{2}(f), \ldots, H_{q}(f)$ which may depend on $f \in \mathcal{F}$ ? The main aim of this article is to give an affirmative answer to this question. Namely, we prove the following result, which generalizes both Theorems A and B.

THEOREM 1.1. Let $\mathcal{F}$ be a family of meromorphic mappings of a domain $D$ in $\mathbb{C}^{n}$ into $\mathbb{P}^{N}(\mathbb{C})$. Suppose that for each $f \in \mathcal{F}$ there exist $q \geq 2 N+1$ moving hypersurfaces $H_{1}(f), H_{2}(f), \ldots, H_{q}(f)$ in $\mathbb{P}^{N}(\mathbb{C})$ such that the following three conditions are satisfied.

(i) For each $1 \leq k \leq q$, the coefficients of the homogeneous polynomials $Q_{k}(f)$ which define the $H_{k}(f)$ are bounded above uniformly for all $f$ in $\mathcal{F}$ on compact subsets of $D$, and for any sequence $\left\{f^{(p)}\right\} \subset \mathcal{F}$, there exists $z \in D$ (which may depend on the sequence) such that

$$
\inf _{p \in \mathbb{N}}\left\{D\left(Q_{1}\left(f^{(p)}\right), \ldots, Q_{q}\left(f^{(p)}\right)\right)(z)\right\}>0 .
$$

(ii) For any fixed compact subset $K$ of $D$, the $2(n-1)$-dimensional Lebesgue areas of $f^{-1}\left(H_{i}(f)\right) \cap K \quad(1 \leq i \leq N+1)$ counting multiplicities are bounded above for all $f$ in $\mathcal{F}$ (in particular, $f(D) \not \subset H_{i}(f) \quad(1 \leq i \leq$ $N+1)$ ). 
(iii) There exists a closed subset $S$ of $D$ with $\Lambda^{2 n-1}(S)=0$ such that, for any fixed compact subset $K$ of $D-S$, the $2(n-1)$-dimensional Lebesgue areas of $f^{-1}\left(H_{i}(f)\right) \cap K(N+2 \leq i \leq q)$ ignoring multiplicities are bounded above for all $f$ in $\mathcal{F}$.

Then $\mathcal{F}$ is a meromorphically normal family on $D$.

In the special case of a family of holomorphic mappings, we get the following with the same proof methods.

THEOREM 1.2. Let $\mathcal{F}$ be a family of holomorphic mappings of a domain $D$ in $\mathbb{C}^{n}$ into $\mathbb{P}^{N}(\mathbb{C})$. Suppose that for each $f \in \mathcal{F}$ there exist $q \geq 2 N+1$ moving hypersurfaces $H_{1}(f), H_{2}(f), \ldots, H_{q}(f)$ in $\mathbb{P}^{N}(\mathbb{C})$ such that the following three conditions are satisfied.

(i) For each $1 \leq k \leq q$, the coefficients of the homogeneous polynomials $Q_{k}(f)$ which define the $H_{k}(f)$ are bounded above uniformly for all $f$ in $\mathcal{F}$ on compact subsets of $D$, and for any sequence $\left\{f^{(p)}\right\} \subset \mathcal{F}$, there exists $z \in D$ (which may depend on the sequence) such that

$$
\inf _{p \in \mathbb{N}}\left\{D\left(Q_{1}\left(f^{(p)}\right), \ldots, Q_{q}\left(f^{(p)}\right)\right)(z)\right\}>0
$$

(ii) Assume that $f(D) \cap H_{i}(f)=\emptyset(1 \leq i \leq N+1)$ for any $f \in F$.

(iii) There exists a closed subset $S$ of $D$ with $\Lambda^{2 n-1}(S)=0$ such that, for any fixed compact subset $K$ of $D-S$, the $2(n-1)$-dimensional Lebesgue areas of $f^{-1}\left(H_{i}(f)\right) \cap K(N+2 \leq i \leq q)$ ignoring multiplicities are bounded above for all $f$ in $\mathcal{F}$.

Then $\mathcal{F}$ is a holomorphically normal family on $D$.

REMARK 1.1. Several examples in [21] show that the conditions in Theorem 1.1(i)-(iii) and Theorem 1.2(i)-(iii) cannot be omitted.

We also generalize several results of Tu from [20]-[22] which allow us not to take into account at all the components of $f^{-1}\left(H_{i}(f)\right)$ of high order, as follows.

Theorem 1.3 generalizes [22, Theorem 2.1] from the case of moving hyperplanes which are independent of $f$ to moving hypersurfaces which may depend on $f$. (In fact, observe that for moving hyperplanes the condition $H_{1}, \ldots, H_{q}$ in $\widetilde{\mathcal{S}}\left(\left\{T_{i}\right\}_{i=0}^{N}\right)$ is satisfied by taking $T_{0}, \ldots, T_{N}$ any (fixed or moving) $N+1$ hyperplanes in general position.) 
THEOREM 1.3. Let $\mathcal{F}$ be a family of holomorphic mappings of a domain $D$ in $\mathbb{C}^{n}$ into $\mathbb{P}^{N}(\mathbb{C})$. Let $q \geq 2 N+1$ be a positive integer. Let $m_{1}, \ldots, m_{q}$ be positive integers or $\infty$ such that

$$
\sum_{j=1}^{q}\left(1-\frac{N}{m_{j}}\right)>N+1 .
$$

Suppose that for each $f \in \mathcal{F}$ there exist $N+1$ moving hypersurfaces $T_{0}(f)$, $\ldots, T_{N}(f)$ in $\mathbb{P}^{N}(\mathbb{C})$ of common degree and that there exist $q$ moving hypersurfaces $H_{1}(f), \ldots, H_{q}(f)$ in $\widetilde{\mathcal{S}}\left(\left\{T_{i}(f)\right\}_{i=0}^{N}\right)$ such that the following conditions are satisfied.

(i) For each $0 \leq i \leq N$, the coefficients of the homogeneous polynomials $P_{i}(f)$ which define the $T_{i}(f)$ are bounded above uniformly for all $f$ in $\mathcal{F}$ on compact subsets of $D$; for all $1 \leq j \leq q$, the coefficients $b_{i j}(f)$ of the linear combinations of the $P_{i}(f), i=0, \ldots, N$ which define the homogeneous polynomials $Q_{j}(f)$ which define the $H_{j}(f)$ are bounded above uniformly for all $f$ in $\mathcal{F}$ on compact subsets of $D$; and for any fixed $z \in D$,

$$
\inf \left\{D\left(Q_{1}(f), \ldots, Q_{q}(f)\right)(z): f \in \mathcal{F}\right\}>0 .
$$

(ii) Assume that $f$ intersects $H_{j}(f)$ with multiplicity at least $m_{j}$ for each $1 \leq j \leq q$. (See Section 2.6 for the necessary definitions.)

Then $\mathcal{F}$ is a holomorphically normal family on $D$.

The following theorem generalizes [21, Theorem 1] from the case of fixed hyperplanes to moving hypersurfaces. (In fact, observe that for hyperplanes the condition $H_{1}(f), \ldots, H_{q}(f)$ in $\widetilde{\mathcal{S}}\left(\left\{T_{i}(f)\right\}_{i=0}^{N}\right)$ is satisfied by taking $T_{0}(f)$, $\ldots, T_{N}(f)$ any $N+1$ hyperplanes in general position.)

THEOREM 1.4. Let $\mathcal{F}$ be a family of meromorphic mappings of a domain $D$ in $\mathbb{C}^{n}$ into $\mathbb{P}^{N}(\mathbb{C})$. Let $q \geq 2 N+1$ be a positive integer. Suppose that for each $f \in \mathcal{F}$ there exist $N+1$ moving hypersurfaces $T_{0}(f), \ldots, T_{N}(f)$ in $\mathbb{P}^{N}(\mathbb{C})$ of common degree and that there exist $q$ moving hypersurfaces $H_{1}(f), \ldots, H_{q}(f)$ in $\widetilde{\mathcal{S}}\left(\left\{T_{i}(f)\right\}_{i=0}^{N}\right)$ such that the following conditions are satisfied.

(i) For each $0 \leq i \leq N$, the coefficients of the homogeneous polynomials $P_{i}(f)$ which define the $T_{i}(f)$ are bounded above uniformly for all $f$ in $\mathcal{F}$ on compact subsets of $D$; for all $1 \leq j \leq q$, the coefficients $b_{i j}(f)$ of the linear combinations of the $P_{i}(f), i=0, \ldots, N$ which define the homogeneous polynomials $Q_{j}(f)$ which define the $H_{j}(f)$ are bounded above uniformly for 
all $f$ in $\mathcal{F}$ on compact subsets of $D$; and for any sequence $\left\{f^{(p)}\right\} \subset \mathcal{F}$, there exists $z \in D$ (which may depend on the sequence) such that

$$
\inf _{p \in \mathbb{N}}\left\{D\left(Q_{1}\left(f^{(p)}\right), \ldots, Q_{q}\left(f^{(p)}\right)\right)(z)\right\}>0 .
$$

(ii) For any fixed compact $K$ of $D$, the $2(n-1)$-dimensional Lebesgue areas of $f^{-1}\left(H_{k}(f)\right) \cap K(1 \leq k \leq N+1)$ counting multiplicities are bounded above for all $f \in \mathcal{F}$ (in particular, $f(D) \not \subset H_{k}(f)(1 \leq k \leq N+1)$ ).

(iii) There exists a closed subset $S$ of $D$ with $\Lambda^{2 n-1}(S)=0$ such that for any fixed compact subset $K$ of $D-S$, the $2(n-1)$-dimensional Lebesgue areas of

$$
\left\{z \in \operatorname{Supp} \nu\left(f, H_{k}(f)\right) \mid \nu\left(f, H_{k}(f)\right)(z)<m_{k}\right\} \cap K \quad(N+2 \leq k \leq q)
$$

ignoring multiplicities for all $f \in \mathcal{F}$ are bounded above, where $\left\{m_{k}\right\}_{k=N+2}^{q}$ are fixed positive integers or $\infty$ with

$$
\sum_{k=N+2}^{q} \frac{1}{m_{k}}<\frac{q-(N+1)}{N} .
$$

Then $\mathcal{F}$ is a meromorphically normal family on $D$.

The following theorem generalizes [20, Theorem 1] from the case of fixed hyperplanes to moving hypersurfaces.

THEOREM 1.5. Let $\mathcal{F}$ be a family of holomorphic mappings of a domain $D$ in $\mathbb{C}^{n}$ into $\mathbb{P}^{N}(\mathbb{C})$. Let $q \geq 2 N+1$ be a positive integer. Suppose that for each $f \in \mathcal{F}$ there exist $N+1$ moving hypersurfaces $T_{0}(f), \ldots, T_{N}(f)$ in $\mathbb{P}^{N}(\mathbb{C})$ of common degree and that there exist $q$ moving hypersurfaces $H_{1}(f), \ldots, H_{q}(f)$ in $\widetilde{\mathcal{S}}\left(\left\{T_{i}(f)\right\}_{i=0}^{N}\right)$ such that the following conditions are satisfied.

(i) For each $0 \leq i \leq N$, the coefficients of the homogeneous polynomials $P_{i}(f)$ which define the $T_{i}(f)$ are bounded above uniformly for all $f$ in $\mathcal{F}$ on compact subsets of $D$; for all $1 \leq j \leq q$, the coefficients $b_{i j}(f)$ of the linear combinations of the $P_{i}(f), i=0, \ldots, N$ which define the homogeneous polynomials $Q_{j}(f)$ which define the $H_{j}(f)$ are bounded above uniformly for all $f$ in $\mathcal{F}$ on compact subsets of $D$; and for any sequence $\left\{f^{(p)}\right\} \subset \mathcal{F}$, there exists $z \in D$ (which may depend on the sequence) such that

$$
\inf _{p \in \mathbb{N}}\left\{D\left(Q_{1}\left(f^{(p)}\right), \ldots, Q_{q}\left(f^{(p)}\right)\right)(z)\right\}>0 .
$$


(ii) Assume that $f(D) \cap H_{i}(f)=\emptyset(1 \leq i \leq N+1)$ for any $f \in \mathcal{F}$.

(iii) There exists a closed subset $S$ of $D$ with $\Lambda^{2 n-1}(S)=0$ such that, for any fixed compact subset $K$ of $D-S$, the $2(n-1)$-dimensional Lebesgue areas of

$$
\left\{z \in \operatorname{Supp} \nu\left(f, H_{k}(f)\right) \mid \nu\left(f, H_{k}(f)\right)(z)<m_{k}\right\} \cap K \quad(N+2 \leq k \leq q)
$$

ignoring multiplicities for all $f$ in $\mathcal{F}$ are bounded above, where $\left\{m_{k}\right\}_{k=N+2}^{q}$ are fixed positive integers and may be $\infty$ with

$$
\sum_{k=N+2}^{q} \frac{1}{m_{k}}<\frac{q-(N+1)}{N} .
$$

Then $\mathcal{F}$ is a holomorphically normal family on $D$.

Let us finally give some comments on our proof methods.

The proofs of Theorems 1.1 and 1.2 are obtained by generalizing ideas, which have been used by Mai, Thai, and Trang in [8] to prove Theorem A, to moving targets, which presents several highly nontrivial technical difficulties. Among others, for a sequence of moving targets $H\left(f^{(p)}\right)$ which at the same time may depend of the meromorphic maps $f^{(p)}: D \rightarrow \mathbb{P}^{N}(\mathbb{C})$, obtaining a subsequence which converges locally uniformly on $D$ is much more difficult than for fixed targets. (Among other difficulties, we cannot normalize the coefficients to have norm equal to 1 everywhere as we can for fixed targets.) This is obtained in Lemma 3.6, after having proved in Lemma 3.5 that the condition $D\left(Q_{1}, \ldots, Q_{q}\right)>\delta>0$ forces a uniform bound, only in terms of $\delta$, on the degrees of the $Q_{i}, 1 \leq i \leq q$. (In fact the latter result fixes also a gap in [8] even for the case of fixed targets.)

The proofs of Theorems 1.3, 1.4, and 1.5 are obtained by combining methods used by $\mathrm{Tu}$ in [20] and [21] and by $\mathrm{Tu}$ and $\mathrm{Li}$ in [22] with the methods which we developed to prove our first two theorems. However, in order to apply the techniques used by $\mathrm{Tu}$ and by $\mathrm{Tu}$ and $\mathrm{Li}$ for the case of hyperplanes, we still need that, for every meromorphic map $f^{(p)}: D \rightarrow$ $\mathbb{P}^{N}(\mathbb{C})$, the $Q_{1}\left(f^{(p)}\right), \ldots, Q_{q}\left(f^{(p)}\right)$ are still in a linear system given by $N+1$ such maps $P_{0}\left(f^{(p)}\right), \ldots, P_{N}\left(f^{(p)}\right)$. Lemmas $3.11-3.14$ adapt our techniques to this situation. (For example, Lemma 3.14 is an adaptation of Lemma 3.6.) 


\section{$\S 2$. Basic notions}

\subsection{Meromorphic mappings}

Let A be a nonempty open subset of a domain $D$ in $\mathbb{C}^{n}$ such that $S=$ $D-A$ is an analytic set in $D$. Let $f: A \rightarrow \mathbb{P}^{N}(\mathbb{C})$ be a holomorphic mapping. Let $U$ be a nonempty connected open subset of $D$. A holomorphic mapping $\tilde{f} \not \equiv 0$ from $U$ into $\mathbb{C}^{N+1}$ is said to be a representation of $f$ on $U$ if $f(z)=$ $\rho(\tilde{f}(z))$ for all $z \in U \cap A-\tilde{f}^{-1}(0)$, where $\rho: \mathbb{C}^{N+1}-\{0\} \rightarrow \mathbb{P}^{N}(\mathbb{C})$ is the canonical projection. A holomorphic mapping $f: A \rightarrow \mathbb{P}^{N}(\mathbb{C})$ is said to be a meromorphic mapping from $D$ into $\mathbb{P}^{N}(\mathbb{C})$ if for each $z \in D$ there exists a representation of $f$ on some neighborhood of $z$ in $D$.

\subsection{Admissible representations}

Let $f$ be a meromorphic mapping of a domain $D$ in $\mathbb{C}^{n}$ into $\mathbb{P}^{N}(\mathbb{C})$. Then for any $a \in D, f$ always has an admissible representation $\tilde{f}(z)=$ $\left(f_{0}(z), f_{1}(z), \ldots, f_{N}(z)\right)$ on some neighborhood $U$ of $a$ in $D$, which means that each $f_{i}(z)$ is a holomorphic function on $U$ and that $f(z)=\left(f_{0}(z)\right.$ : $\left.f_{1}(z): \cdots: f_{N}(z)\right)$ outside the analytic set $I(f):=\left\{z \in U: f_{0}(z)=f_{1}(z)=\right.$ $\left.\cdots=f_{N}(z)=0\right\}$ of codimension $\geq 2$.

\subsection{Moving hypersurfaces in general position}

Let $D$ be a domain in $\mathbb{C}^{n}$. Denote by $\mathcal{H}_{D}$ the ring of all holomorphic functions on $D$, and denote by $\widetilde{\mathcal{H}}_{D}\left[\omega_{0}, \ldots, \omega_{N}\right]$ the set of all homogeneous polynomials $Q \in \mathcal{H}_{D}\left[\omega_{0}, \ldots, \omega_{N}\right]$ such that the coefficients of $Q$ are not all identically zero. Each element of $\widetilde{\mathcal{H}}_{D}\left[\omega_{0}, \ldots, \omega_{N}\right]$ is said to be a moving hypersurface in $\mathbb{P}^{N}(\mathbb{C})$.

Let $Q$ be a moving hypersurface of degree $d \geq 1$. Denote by $Q(z)$ the homogeneous polynomial over $\mathbb{C}^{N+1}$ obtained by evaluating the coefficients of $Q$ in a specific point $z \in D$. We remark that for generic $z \in D$ this is a nonzero homogeneous polynomial with coefficients in $\mathbb{C}$. The hypersurface $H$ given by $H(z):=\left\{w \in \mathbb{C}^{N+1}: Q(z)(w)=0\right\}$ (for generic $z \in D$ ) is also called a moving hypersurface in $\mathbb{P}^{N}(\mathbb{C})$, which is defined by $Q$. In this article, we identify $Q$ with $H$ if no confusion arises.

We say that moving hypersurfaces $\left\{Q_{j}\right\}_{j=1}^{q}$ of degree $d_{j}(q \geq N+1)$ in $\mathbb{P}^{N}(\mathbb{C})$ are located in (weakly) general position if there exists $z \in D$ such that, for any $1 \leq j_{0}<\cdots<j_{N} \leq q$, the system of equations

$$
\left\{\begin{array}{l}
Q_{j_{i}}(z)\left(\omega_{0}, \ldots, \omega_{N}\right)=0 \\
0 \leq i \leq N
\end{array}\right.
$$


has only the trivial solution $\omega=(0, \ldots, 0)$ in $\mathbb{C}^{N+1}$. This is equivalent to

$$
\begin{aligned}
& D\left(Q_{1}, \ldots, Q_{q}\right)(z) \\
& :=\prod_{1 \leq j_{0}<\cdots<j_{N} \leq q} \inf _{\|\omega\|=1}\left(\left|Q_{j_{0}}(z)(\omega)\right|^{2}+\cdots+\left|Q_{j_{N}}(z)(\omega)\right|^{2}\right)>0
\end{aligned}
$$

where $Q_{j}(z)(\omega)=\sum_{|I|=d_{j}} a_{j I}(z) \cdot \omega^{I}$ and $\|\omega\|=\left(\sum\left|\omega_{j}\right|^{2}\right)^{1 / 2}$.

\subsection{Divisors}

Let $D$ be a domain in $\mathbb{C}^{n}$, and let $f$ be a nonidentically zero holomorphic function on $D$. For a point $a=\left(a_{1}, a_{2}, \ldots, a_{n}\right) \in D$ we expand $f$ as a compactly convergent series

$$
f\left(u_{1}+a_{1}, \ldots, u_{n}+a_{n}\right)=\sum_{m=0}^{\infty} P_{m}\left(u_{1}, \ldots, u_{n}\right)
$$

on a neighborhood of $a$, where $P_{m}$ is either identically zero or a homogeneous polynomial of degree $m$. The number

$$
\nu_{f}(a):=\min \left\{m ; P_{m}(u) \not \equiv 0\right\}
$$

is said to be the zero multiplicity of $f$ at $a$. By definition, a divisor on $D$ is an integer-valued function $\nu$ on $D$ such that for every $a \in D$ there are holomorphic functions $g(z)(\not \equiv 0)$ and $h(z)(\not \equiv 0)$ on a neighborhood $U$ of $a$ with $\nu(z)=\nu_{g}(z)-\nu_{h}(z)$ on $U$. We define the support of the divisor $\nu$ on $D$ by

$$
\operatorname{Supp} \nu:=\overline{\{z \in D: \nu(z) \neq 0\}} .
$$

We denote $\mathcal{D}^{+}(D)=\{\nu$ : a nonnegative divisor on $D\}$.

Let $f$ be a meromorphic mapping from a domain $D$ into $\mathbb{P}^{N}(\mathbb{C})$. For each homogeneous polynomial $Q \in \widetilde{\mathcal{H}}_{D}\left[\omega_{0}, \ldots, \omega_{N}\right]$, we define the divisor $\nu(f, Q)$ on $D$ as follows. For each $a \in D$, let $\widetilde{f}=\left(f_{0}, \ldots, f_{N}\right)$ be an admissible representation of $f$ in a neighborhood $U$ of $a$. Then we put

$$
\nu(f, Q)(a):=\nu_{Q(\tilde{f})}(a)
$$

where $Q(\tilde{f}):=Q\left(f_{0}, \ldots, f_{N}\right)$.

Let $H$ be a moving hypersurface which is defined by the homogeneous polynomial $Q \in \widetilde{\mathcal{H}}_{D}\left[\omega_{0}, \ldots, \omega_{N}\right]$, and let $f$ be a meromorphic mapping of $D$ into $\mathbb{P}^{N}(\mathbb{C})$. As above, we define the divisor $\nu(f, H)(z):=\nu(f, Q)(z)$. Obvi- 
ously, $\operatorname{Supp} \nu(f, H)$ is either an empty set or a pure $(n-1)$-dimensional analytic set in $D$ if $f(D) \not \subset H$ (i.e., $Q(\tilde{f}) \not \equiv 0$ on $U)$. We define $\nu(f, H)=\infty$ on $D$ and $\operatorname{Supp} \nu(f, H)=D$ if $f(D) \subset H$. Sometimes we identify $f^{-1}(H)$ with the divisor $\nu(f, H)$ on $D$. We can rewrite $\nu(f, H)$ as the formal sum $\nu(f, H)=$ $\sum_{i \in I} n_{i} X_{i}$, where $X_{i}$ are the irreducible components of $\operatorname{Supp} \nu(f, H)$ and $n_{i}$ are the constants $\nu(f, H)(z)$ on $X_{i} \cap \operatorname{Reg}(\operatorname{Supp} \nu(f, H))$, where $\operatorname{Reg}()$ denotes the set of all the regular points.

We say that the meromorphic mapping $f$ intersects $H$ with multiplicity at least $m$ on $D$ if $\nu(f, H)(z) \geq m$ for all $z \in \operatorname{Supp} \nu(f, H)$ and, in particular, that $f$ intersects $H$ with multiplicity $\infty$ on $D$ if $f(D) \subset H$ or $f(D) \cap H=\emptyset$.

\subsection{Meromorphically normal families}

Let $D$ be a domain in $\mathbb{C}^{n}$.

(i) (See [1].) Let $\mathcal{F}$ be a family of holomorphic mappings of $D$ into a compact complex manifold $M$. This family $\mathcal{F}$ is said to be a (holomorphically) normal family on $D$ if any sequence in $\mathcal{F}$ contains a subsequence which converges uniformly on compact subsets of $D$ to a holomorphic mapping of $D$ into $M$.

(ii) (See [4].) A sequence $\left\{f^{(p)}\right\}$ of meromorphic mappings from $D$ into $\mathbb{P}^{N}(\mathbb{C})$ is said to converge meromorphically on $D$ to a meromorphic mapping $f$ if and only if, for any $z \in D$, each $f^{(p)}$ has an admissible representation

$$
\tilde{f}^{(p)}=\left(f_{0}^{(p)}: f_{1}^{(p)}: \cdots: f_{N}^{(p)}\right)
$$

on some fixed neighborhood $U$ of $z$ such that $\left\{f_{i}^{(p)}\right\}_{p=1}^{\infty}$ converges uniformly on compact subsets of $U$ to a holomorphic function $f_{i}(0 \leq i \leq N)$ on $U$ with the property that $\tilde{f}=\left(f_{0}: f_{1}: \cdots: f_{N}\right)$ is a representation of $f$ on $U$ (not necessarily an admissible one!).

(iii) (See [4].) Let $\mathcal{F}$ be a family of meromorphic mappings of $D$ into $\mathbb{P}^{N}(\mathbb{C})$. One may call $\mathcal{F}$ a meromorphically normal family on $D$ if any sequence in $\mathcal{F}$ has a meromorphically convergent subsequence on $D$.

(iv) (See [15].) Let $\left\{\nu_{i}\right\}$ be a sequence of nonnegative divisors on $D$. It is said to converge to a nonnegative divisor $\nu$ on $D$ if and only if any $a \in D$ has a neighborhood $U$ such that there exist holomorphic functions $h_{i}(\not \equiv 0)$ and $h(\not \equiv 0)$ on $U$ such that $\nu_{i}=\nu_{h_{i}}, \nu=\nu_{h}$, and $\left\{h_{i}\right\}$ converges compactly to $h$ on $U$.

(v) (See [15].) Let $\left\{A_{i}\right\}$ be a sequence of closed subsets of $D$. It is said to converge to a closed subset $A$ of $D$ if and only if $A$ coincides with the set of all $z$ such that every neighborhood $U$ of $z$ intersects $A_{i}$ for all but 
finitely many $i$ and, simultaneously, with the set of all $z$ such that every $U$ intersects $A_{i}$ for infinitely many $i$.

\subsection{Other notation}

Let $P_{0}, \ldots, P_{N}$ be $N+1$ homogeneous polynomials of common degree in $\mathbb{C}\left[\omega_{0}, \ldots, \omega_{N}\right]$. Denote by $\mathcal{S}\left(\left\{P_{i}\right\}_{i=0}^{N}\right)$ the set of all homogeneous polynomials $Q=\sum_{i=0}^{N} b_{i} P_{i}\left(b_{i} \in \mathbb{C}\right)$.

Let $\left\{Q_{j}:=\sum_{i=0}^{N} b_{j i} P_{i}\right\}_{j=1}^{q}$ be $q(q \geq N+1)$ homogeneous polynomials in $\mathcal{S}\left(\left\{P_{i}\right\}_{i=0}^{N}\right)$. We say that $\left\{Q_{j}\right\}_{j=1}^{q}$ are located in general position in $\mathcal{S}\left(\left\{P_{i}\right\}_{i=0}^{N}\right)$ if

$$
\forall 1 \leq j_{0}<\cdots<j_{N} \leq q, \quad \operatorname{det}\left(b_{j_{k} i}\right)_{0 \leq k, i \leq N} \neq 0 .
$$

Let $T_{0}, \ldots, T_{N}$ be hypersurfaces in $\mathbb{P}^{N}(\mathbb{C})$ of common degree, where $T_{i}$ is defined by the (not zero) polynomial $P_{i}(0 \leq i \leq N)$. Denote by $\widetilde{\mathcal{S}}\left(\left\{T_{i}\right\}_{i=0}^{N}\right)$ the set of all hypersurfaces in $\mathbb{P}^{N}(\mathbb{C})$ which are defined by $Q \in \mathcal{S}\left(\left\{P_{i}\right\}_{i=0}^{N}\right)$ with $Q$ not zero.

Let $P_{0}, \ldots, P_{N}$ be $N+1$ homogeneous polynomials of common degree in $\widetilde{\mathcal{H}}_{D}\left[\omega_{0}, \ldots, \omega_{N}\right]$. Denote by $\widetilde{\mathcal{S}}\left(\left\{P_{i}\right\}_{i=0}^{N}\right)$ the set of all homogeneous not identically zero polynomials $Q=\sum_{i=0}^{N} b_{i} P_{i}\left(b_{i} \in \mathcal{H}_{D}\right)$.

Let $T_{0}, \ldots, T_{N}$ be moving hypersurfaces in $\mathbb{P}^{N}(\mathbb{C})$ of common degree, where $T_{i}$ is defined by the (not identically zero) polynomial $P_{i}(0 \leq i \leq N)$. Denote by $\widetilde{\mathcal{S}}\left(\left\{T_{i}\right\}_{i=0}^{N}\right)$ the set of all moving hypersurfaces in $\mathbb{P}^{N}(\mathbb{C})$ which are defined by $Q \in \widetilde{\mathcal{S}}\left(\left\{P_{i}\right\}_{i=0}^{N}\right)$.

Denote by $\operatorname{Hol}(X, Y)$ the set of all holomorphic mappings from a complex space $X$ to a complex space $Y$.

For each $x \in \mathbb{C}^{n}$ and $R>0$, we set $B(x, R)=\left\{z \in \mathbb{C}^{n}:\|z-x\|<R\right\}$ and $B(R)=B(0, R)$.

Let $\Lambda^{d}(S)$ denote the real $d$-dimensional Hausdorff measure of $S \subset \mathbb{C}^{n}$. For a formal $\mathbb{Z}$-linear combination $X=\sum_{i \in I} n_{i} X_{i}$ of analytic subsets $X_{i} \subset$ $\mathbb{C}^{n}$ and for a subset $E \subset \mathbb{C}^{n}$, we call $\sum_{i \in I} \Lambda^{d}\left(X_{i} \cap E\right)$ (resp., $\sum_{i \in I} n_{i} \Lambda^{d}\left(X_{i} \cap\right.$ $E)$ ) the $d$-dimensional Lebesgue area of $X \cap E$, ignoring multiplicities (resp., with counting multiplicities).

Finally, we list some facts on Hausdorff measures for later use which can be found, for example, in [2].

Lemma 2.1 ([2, pp. 351-352 and 299-300]). Let $B:=B(x, R) \subset \mathbb{C}^{n}$, and let $S \subset B$ be a closed subset with $\Lambda^{2 n-1}(S)=0$. Let $h: B \rightarrow \mathbb{C}$ be a holomorphic function, let $h \not \equiv 0$, and let $S_{1}:=S \cup\{h=0\}$. Let $z_{0} \in B$. Then we have the following. 
(a) For almost every complex line $l:=\left\{z_{0}+z \cdot u, z \in \mathbb{C}\right\}$ passing through $z_{0}$, we have $\Lambda^{1}\left(S_{1} \cap l\right)=0$.

(b) Let $r_{0}:=\operatorname{dist}\left(z_{0}, \partial B\right)$, and for every $\left.r \in\right] 0, r_{0}\left[\right.$, let $\mathcal{C}_{r}:=\left\{z_{0}+r \cdot e^{i \theta} \cdot u\right.$ : $\theta \in[0,2 \pi]\} \subset l$. If $\Lambda^{1}\left(S_{1} \cap l\right)=0$, then the subset of the $r$ such that $\mathcal{C}_{r} \cap S_{1} \neq \emptyset$ is nowhere dense in the interval $] 0, r_{0}[$.

Corollary 2.2. With the notations of Lemma 2.1, the set $B \backslash S_{1}$ is pathwise connected.

Proof. This is an immediate consequence of Lemma 2.1 and of the fact that $B \backslash S_{1} \subset B$ is an open subset.

\section{§3. Lemmas}

Lemma 3.1 ([15, Theorem 2.24]). A sequence $\left\{\nu_{i}\right\}$ of nonnegative divisors on a domain $D$ in $\mathbb{C}^{n}$ is normal in the sense of the convergence of divisors on $D$ if and only if the $2(n-1)$-dimensional Lebesgue areas of $\nu_{i} \cap E(i \geq 1)$ with counting multiplicities are bounded above for any fixed compact set $E$ of $D$.

LEMMA 3.2 ([15, Theorem 4.10]). If a sequence $\left\{\nu_{i}\right\}$ converges to $\nu$ in $\mathcal{D}^{+}(B(R))$, then $\left\{\operatorname{supp} \nu_{i}\right\}$ converges to $\operatorname{supp} \nu$ (in the sense of closed subsets of $D)$.

Lemma 3.3 ([15, Proposition 4.12]). Let $\left\{N_{i}\right\}$ be a sequence of pure $(n-1)$-dimensional analytic subsets of a domain $D$ in $\mathbb{C}^{n}$. If the $2(n-1)$ dimensional Lebesgue areas of $N_{i} \cap K$ ignoring multiplicities $(i=1,2, \ldots)$ are bounded above for any fixed compact subset $K$ of $D$, then $\left\{N_{i}\right\}$ is normal in the sense of the convergence of closed subsets in $D$.

Lemma 3.4 ([15, Proposition 4.11]). Let $\left\{N_{i}\right\}$ be a sequence of pure $(n-1)$-dimensional analytic subsets of a domain $D$ in $\mathbb{C}^{n}$. Assume that the $2(n-1)$-dimensional Lebesgue areas of $N_{i} \cap K$ ignoring multiplicities $(i=1,2, \ldots)$ are bounded above for any fixed compact subset $K$ of $D$ and that $\left\{N_{i}\right\}$ converges to $N$ as a sequence of closed subsets of $D$. Then $N$ is either empty or a pure $(n-1)$-dimensional analytic subset of $D$.

LEMma 3.5. Let natural numbers $N$ and $q \geq N+1$ be fixed. Then for each $\delta>0$, there exists $M(\delta)=M(\delta, N, q)>0$ such that the following is satisfied.

For any homogeneous polynomials $Q_{1}, \ldots, Q_{q}$ on $\mathbb{C}^{N+1}$ with complex coefficients with norms bounded above by 1 such that $D\left(Q_{1}, \ldots, Q_{q}\right)>\delta$, we have $\max \left\{\operatorname{deg} Q_{1}, \ldots, \operatorname{deg} Q_{q}\right\}<M(\delta)$. 
Proof. First of all, we make the following three remarks.

(i) Let $Q(\omega)$ be a homogeneous polynomial on $\mathbb{C}^{N+1}$ such that

$$
Q(\omega)=\sum_{|\alpha|=d} a_{\alpha} \omega^{\alpha}
$$

where $\left|a_{\alpha}\right| \leq 1$. Then

$$
|Q(\omega)| \leq \sum_{|\alpha|=d}\left|\omega_{0}\right|^{\alpha_{0}} \cdots\left|\omega_{N}\right|^{\alpha_{N}} \leq(d+1)^{N+1} r^{d}
$$

when $\left|\omega_{k}\right| \leq r \forall 0 \leq k \leq N$.

We set

$$
M_{0}=\sup _{d \in \mathbb{Z}^{+}}(d+1)^{N+1}\left(\frac{1}{\sqrt{N+1}}\right)^{d} .
$$

Since $\lim _{d \longrightarrow+\infty}(d+1)^{N+1}(1 / \sqrt{N+1})^{d}=0$, it implies that $M_{0}<+\infty$.

(ii) Let $Q_{0}, \ldots, Q_{N}$ be homogeneous polynomials on $\mathbb{C}^{N+1}$ such that the norms of their complex coefficients are bounded above by 1 and $D\left(Q_{0}, \ldots\right.$, $\left.Q_{N}\right)>0$. We choose $\omega^{(0)}=(1 / \sqrt{N+1}, \ldots, 1 / \sqrt{N+1}) \in \mathbb{C}^{N+1}$. Then $\left\|\omega^{(0)}\right\|=1$. By (i), we have

$$
D\left(Q_{0}, \ldots, Q_{N}\right) \leq(N+1) M_{0}^{2}<+\infty .
$$

(iii) Since $\lim _{x \rightarrow \infty}(1-(1 / x))^{x}=1 / e$, we have $\left(e(1-(1 / x))^{x}\right) / 2<1$ for $x$ big enough. Therefore,

$$
\lim _{x \rightarrow \infty}\left(x^{2}+1\right)^{N+1}\left(1-\frac{1}{x}\right)^{x^{2}}=\lim _{x \rightarrow \infty}\left(\frac{e\left(1-\frac{1}{x}\right)^{x}}{2}\right)^{x} \frac{\left(x^{2}+1\right)^{N+1}}{\left(\frac{e}{2}\right)^{x}}=0 .
$$

We now come back to the proof of Lemma 3.5, and we consider the following two cases.

Case 1: $q=N+1$.

Assume that such a constant $M(\delta)=M(\delta, N, N+1)$ does not exist. Then there exist homogeneous polynomials $Q_{0}^{(j)}, \ldots, Q_{N}^{(j)}(j \geq 1)$ with coefficients being bounded above by 1 such that

$$
\begin{aligned}
\inf \left\{D\left(Q_{0}^{(j)}, \ldots, Q_{N}^{(j)}\right): j \geq 1\right\}>\delta>0, \\
\lim _{j \rightarrow \infty}\left(\max \left\{\operatorname{deg} Q_{0}^{(j)}, \ldots, \operatorname{deg} Q_{N}^{(j)}\right\}\right)=\infty .
\end{aligned}
$$


Without loss of generality, we may assume that

$$
\begin{aligned}
& \operatorname{deg} Q_{i}^{(j)}=d_{i} \quad \forall 0 \leq i \leq k, \forall j \geq 1, \quad \text { and } \\
& \operatorname{deg} Q_{i}^{(j)}=d_{i}^{(j)} \rightarrow \infty \text { as } j \rightarrow \infty \quad \text { for each } k+1 \leq i \leq N,
\end{aligned}
$$

where $k$ is some integer such that $0 \leq k \leq N-1$.

Since $\operatorname{deg} Q_{i}^{(j)}=d_{i} \forall 0 \leq i \leq k, \forall j \geq 1$, we may assume that, for each $0 \leq$ $i \leq k,\left\{Q_{i}^{(j)}\right\}_{j \geq 1}$ converges uniformly on compact subsets of $\mathbb{C}^{N+1}$ either to a homogeneous polynomial $Q_{i}$ of degree $d_{i}$ with coefficients being bounded above by 1 or to the zero polynomial. Since $0 \leq k \leq N-1$, we have

$$
\bigcap_{i=0}^{k}\left\{H_{i}:=\operatorname{Zero}\left(Q_{i}\right)\right\} \neq \emptyset \text {. }
$$

Hence, there exists $\omega^{(0)} \in \bigcap_{i=0}^{k} H_{i}$ with $\left\|\omega^{(0)}\right\|=1$. We now consider two subcases.

Subcase 1.1. Assume that $r=\max \left\{\left|\omega_{0}^{(0)}\right|, \ldots,\left|\omega_{N}^{(0)}\right|\right\}<1$.

- If $0 \leq i \leq k$, then

$$
\lim _{j \rightarrow \infty} Q_{i}^{(j)}\left(\omega^{(0)}\right)=0
$$

- If $k+1 \leq i \leq N$, then, by (i), we have

$$
\left|Q_{i}^{(j)}\left(\omega^{(0)}\right)\right| \leq\left(d_{i}^{(j)}+1\right)^{N+1} r^{d_{i}^{(j)}}
$$

Since $\lim _{j \rightarrow \infty} d_{i}^{(j)}=\infty$ and $r<1$, it implies that

$$
\lim _{j \rightarrow \infty} Q_{i}^{(j)}\left(\omega^{(0)}\right)=0 \quad \text { for } k+1 \leq i \leq N .
$$

Therefore, we get

$$
\lim _{j \rightarrow \infty} D\left(Q_{0}^{(j)}, \ldots, Q_{N}^{(j)}\right) \leq \lim _{j \rightarrow \infty} \sum_{i=0}^{N}\left|Q_{i}^{(j)}\left(\omega^{(0)}\right)\right|^{2}=0 .
$$

This is a contradiction.

Subcase 1.2. Assume that $\max \left\{\left|\omega_{0}^{(0)}\right|, \ldots,\left|\omega_{N}^{(0)}\right|\right\}=1$.

We may assume that $\omega^{(0)}=(1,0, \ldots, 0)$. Set $\left\{\omega^{(j)}\right\}_{j \geq 1}$ such that

$$
\omega_{0}^{(j)}=1-\frac{1}{\sqrt{d^{(j)}}}, \quad \omega_{1}^{(j)}=\cdots=\omega_{N}^{(j)}=\frac{1}{\sqrt{N}} \sqrt{\frac{2}{\sqrt{d^{(j)}}}-\frac{1}{d^{(j)}}},
$$

where $d^{(j)}=\min _{k+1 \leq i \leq N} d_{i}^{(j)}$. 
- If $0 \leq i \leq k$, then

$$
\lim _{j \rightarrow \infty} Q_{i}^{(j)}\left(\omega^{(j)}\right)=Q_{i}\left(\omega^{(0)}\right)=0 .
$$

- Suppose that $k+1 \leq i \leq N$.

Since $\lim _{j \rightarrow \infty} d^{(j)}=\infty$, there exists $j_{0}$ such that

$$
\max \left\{\left|\omega_{0}^{(j)}\right|, \ldots,\left|\omega_{N}^{(j)}\right|\right\}=\left|\omega_{0}^{(j)}\right|=1-\frac{1}{\sqrt{d^{(j)}}}=r_{j} \quad \text { for any } j>j_{0} .
$$

By (i) and (iii), for each $k+1 \leq i \leq N$, we have

$$
\begin{aligned}
\left|Q_{i}^{(j)}\left(\omega^{(j)}\right)\right| & \leq\left(d_{i}^{(j)}+1\right)^{N+1}\left(1-\frac{1}{\sqrt{d^{(j)}}}\right)^{d_{i}^{(j)}} \\
& \leq\left(d_{i}^{(j)}+1\right)^{N+1}\left(1-\frac{1}{\sqrt{d_{i}^{(j)}}}\right)^{d_{i}^{(j)}} \rightarrow 0 \quad \text { as } j \rightarrow \infty .
\end{aligned}
$$

This is a contradiction by the same argument as above.

Case 2: $q>N+1$.

By (ii) we have

$$
\delta<D\left(Q_{1}, \ldots, Q_{q}\right)=\prod_{1 \leq j_{0}<\cdots<j_{N} \leq q} D\left(Q_{j_{0}}, \ldots, Q_{j_{N}}\right) \leq C D\left(Q_{j_{0}}, \ldots, Q_{j_{N}}\right)
$$

for any set $\left\{j_{0}, \ldots, j_{N}\right\} \subset\{1, \ldots, q\}$, where $C$ is a constant which depends only on $N$ and $q$.

By Case 1, we have

$$
\max \left\{\operatorname{deg} Q_{j_{0}}, \ldots, \operatorname{deg} Q_{j_{N}}\right\}<M(\delta / C, N, N+1)
$$

for any set $\left\{j_{0}, \ldots, j_{N}\right\} \subset\{1, \ldots, q\}$. So if we define

$$
M(\delta, N, q):=M(\delta / C, N, N+1)
$$

(which is well defined since $C$ only depends on $N$ and $q$ ), then we have

$$
\max \left\{\operatorname{deg} Q_{1}, \ldots, \operatorname{deg} Q_{q}\right\}<M(\delta, N, q) .
$$

Lemma 3.6. Let natural numbers $N$ and $q \geq N+1$ be fixed. Let $H_{k}^{(p)}$ $(1 \leq k \leq q, p \geq 1)$ be moving hypersurfaces in $\mathbb{P}^{N}(\mathbb{C})$ such that the following conditions are satisfied. 
(i) For each $1 \leq k \leq q, p \geq 1$, the coefficients of the homogeneous polynomials $Q_{k}^{(p)}$ which define the $H_{k}^{(p)}$ are bounded above uniformly for all $p \geq 1$ on compact subsets of $D$.

(ii) There exists $z_{0} \in D$ such that

$$
\inf _{p \in \mathbb{N}}\left\{D\left(Q_{1}^{(p)}, \ldots, Q_{q}^{(p)}\right)\left(z_{0}\right)\right\}>\delta>0
$$

Then, we have the following.

(a) There exists a subsequence $\left\{j_{p}\right\} \subset \mathbb{N}$ such that for $1 \leq k \leq q, Q_{k}^{\left(j_{p}\right)}$ converge uniformly on compact subsets of $D$ to not identically zero homogeneous polynomials $Q_{k}$ (meaning that the $Q_{k}^{\left(j_{p}\right)}$ and $Q_{k}$ are homogeneous polynomials in $\widetilde{\mathcal{H}}_{D}\left[\omega_{0}, \ldots, \omega_{N}\right]$ of the same degree, and all their coefficients converge uniformly on compact subsets of $D)$. Moreover, we have that $D\left(Q_{1}, \ldots\right.$, $\left.Q_{q}\right)\left(z_{0}\right)>\delta>0$, the hypersurfaces $Q_{1}\left(z_{0}\right), \ldots, Q_{q}\left(z_{0}\right)$ are located in general position, and the moving hypersurfaces $Q_{1}(z), \ldots, Q_{q}(z)$ are located in (weakly) general position.

(b) There exist a subsequence $\left\{j_{p}\right\} \subset \mathbb{N}$ and $r=r(\delta)>0$ such that

$$
\inf \left\{D\left(Q_{1}^{\left(j_{p}\right)}, \ldots, Q_{q}^{\left(j_{p}\right)}\right)(z) \mid p \geq 1\right\}>\frac{\delta}{4}, \quad \forall z \in B\left(z_{0}, r\right) .
$$

Proof. Let $d_{k}^{(p)}=\operatorname{deg} Q_{k}^{(p)}$ be the degree of the nonidentically vanishing homogeneous polynomial $Q_{k}^{(p)}(1 \leq k \leq q, p \geq 1)$. Then we have

$$
Q_{k}^{(p)}(z)(\omega)=\sum_{|I|=d_{k}^{(p)}} a_{k p I}(z) \cdot \omega^{I}
$$

where $I=\left(i_{1}, \ldots, i_{N+1}\right),|I|=i_{1}+\cdots+i_{N+1}$, and $a_{k p I}(z)$ are holomorphic functions which are bounded above uniformly for all $p \geq 1$ on compact subsets of $D$. Since the coefficients of the polynomials $Q_{k}^{\left(j_{p}\right)}$ are bounded above uniformly for all $p \geq 1$ on compact subsets of $D$, there exists $c>0$ such that $\left|a_{k p I}\left(z_{0}\right)\right| \leq c$ for all $k, p, I$. Define homogeneous polynomials

$$
\tilde{Q}_{k}^{(p)}(z)(\omega):=\frac{1}{c} Q_{k}^{(p)}(z)(\omega)
$$

Then the $\tilde{Q}_{k}^{(p)}(z)(\omega)$ satisfy the condition

$$
\inf _{p \in \mathbb{N}}\left\{D\left(\tilde{Q}_{1}^{(p)}, \ldots, \tilde{Q}_{q}^{(p)}\right)\left(z_{0}\right)\right\}>\tilde{\delta}>0
$$


with $\tilde{\delta}:=(1 / c)^{2\left(\begin{array}{c}q \\ N+1\end{array}\right)} \delta$. By Lemma 3.5, we have

$$
\max \left\{\operatorname{deg} \tilde{Q}_{1}^{(p)}\left(z_{0}\right), \ldots, \operatorname{deg} \tilde{Q}_{q}^{(p)}\left(z_{0}\right)\right\}<M(\tilde{\delta}) .
$$

Since by (3.1) none of the homogeneous polynomials $Q_{k}^{(p)}\left(z_{0}\right)(1 \leq k \leq q$, $p \geq 1$ ) can be the zero polynomial, we get that

$$
\max \left\{\operatorname{deg} \tilde{Q}_{1}^{(p)}(z), \ldots, \operatorname{deg} \tilde{Q}_{q}^{(p)}(z)\right\}<M(\tilde{\delta})
$$

for all $z \in D$. So if again

$$
\tilde{Q}_{k}^{(p)}(z)(\omega)=\sum_{|I|=d_{k}^{(p)}} \tilde{a}_{k p I}(z) \cdot \omega^{I}
$$

after passing to a subsequence $\left\{j_{p}\right\} \subset \mathbb{N}$ (which we denote for simplicity again by $\{p\} \subset \mathbb{N})$, we can assume that $d_{k}^{(p)}=d_{k}$ for $1 \leq k \leq q$. So if we still multiply by $c$, we get

$$
Q_{k}^{(p)}(z)(\omega)=\sum_{|I|=d_{k}} a_{k p I}(z) \cdot \omega^{I} .
$$

Now, since the $a_{k p I}(z)$ are locally bounded uniformly for all $p \geq 1$ on $D$, by using Montel's theorem and a standard diagonal argument with respect to an exhaustion of $D$ with compact subsets, after passing to a subsequence $\left\{j_{p}\right\} \subset \mathbb{N}$ (which we denote for simplicity again by $\{p\} \subset \mathbb{N}$ ), we also can assume that $\left\{a_{k p I}(z)\right\}_{p=1}^{\infty}$ converges uniformly on compact subsets of $D$ to $a_{k I}$ for each $k, I$. Denote

$$
Q_{k}(z)(\omega)=\sum_{|I|=d_{k}} a_{k I}(z) \cdot \omega^{I} .
$$

Then

$$
D\left(Q_{1}, \ldots, Q_{q}\right)\left(z_{0}\right) \geq \liminf _{p \longrightarrow \infty} D\left(Q_{1}^{(p)}, \ldots, Q_{q}^{(p)}\right)\left(z_{0}\right)>\delta>0
$$

hence, the hypersurfaces $Q_{1}\left(z_{0}\right), \ldots, Q_{q}\left(z_{0}\right)$ are located in general position, and so the moving hypersurfaces $Q_{1}(z), \ldots, Q_{q}(z)$ are located (weakly) general position (and, in particular, all the $Q_{1}(z), \ldots, Q_{q}(z)$ are not identically zero), which proves (a). 
Moreover, by (3.2), there exists $r=r(\delta)$ such that

$$
D\left(Q_{1}, \ldots, Q_{q}\right)(z)>\frac{\delta}{2}, \quad \forall z \in B\left(z_{0}, r\right) .
$$

Since $\left\{Q_{k}^{(p)}\right\}$ converges uniformly on compact subsets of $D$ to $Q_{k}$, after shrinking $r$ a bit if necessary, there exists $M$ such that

$$
D\left(Q_{1}^{(p)}, \ldots, Q_{q}^{(p)}\right)(z)>\frac{\delta}{4}, \quad \forall z \in B\left(z_{0}, r\right), p>M
$$

which proves (b).

LEMMA 3.7. Let $\left\{f^{(p)}\right\}$ be a sequence of meromorphic mappings of a domain $D$ in $\mathbb{C}^{n}$ into $\mathbb{P}^{N}(\mathbb{C})$, and let $S$ be a closed subset of $D$ with $\Lambda^{2 n-1}(S)=0$. Suppose that $\left\{f^{(p)}\right\}$ meromorphically converges on $D-S$ to a meromorphic mapping $f$ of $D-S$ into $\mathbb{P}^{N}(\mathbb{C})$. Suppose that, for each $f^{(p)}$, there exist $N+1$ moving hypersurfaces $H_{1}\left(f^{(p)}\right), \ldots, H_{N+1}\left(f^{(p)}\right)$ in $\mathbb{P}^{N}(\mathbb{C})$, where the moving hypersurfaces $H_{i}\left(f^{(p)}\right)$ may depend on $f^{(p)}$, such that the following three conditions are satisfied.

(i) For each $1 \leq k \leq N+1$, the coefficients of homogeneous polynomial $Q_{k}\left(f^{(p)}\right)$ which define $H_{k}\left(f^{(p)}\right)$ for all $f^{(p)}$ are bounded above uniformly for all $p \geq 1$ on compact subsets of $D$.

(ii) There exists $z_{0} \in D$ such that

$$
\inf \left\{D\left(Q_{1}\left(f^{(p)}\right), \ldots, Q_{N+1}\left(f^{(p)}\right)\right)\left(z_{0}\right) \mid p \geq 1\right\}>0
$$

(iii) The 2(n-1)-dimensional Lebesgue areas of $\left(f^{(p)}\right)^{-1}\left(H_{k}\left(f^{(p)}\right)\right) \cap K$ $(1 \leq k \leq N+1, p \geq 1)$ counting multiplicities are bounded above for any fixed compact subset $K$ of $D$.

Then we have the following:

(a) $\left\{f^{(p)}\right\}$ has a meromorphically convergent subsequence on $D$, and

(b) if, moreover, $\left\{f^{(p)}\right\}$ is a sequence of holomorphic mappings of a domain $D$ in $\mathbb{C}^{n}$ into $\mathbb{P}^{N}(\mathbb{C})$ and condition (iii) is sharpened to

$$
f^{(p)}(D) \cap H_{k}\left(f^{(p)}\right)=\emptyset \quad(1 \leq k \leq N+1, p \geq 1)
$$

then $\left\{f^{(p)}\right\}$ has a subsequence which converges uniformly on compact subsets of $D$ to a holomorphic mapping of $D$ to $\mathbb{P}^{N}(\mathbb{C})$. 
Proof. By Lemma 3.6 and conditions (i) and (ii), after passing to a subsequence we may assume that for $1 \leq k \leq N+1, Q_{k}\left(f^{(p)}\right)$ converge uniformly on compact subsets of $D$ to $Q_{k}$; in particular, they have common degree $d_{k}$. Moreover, $Q_{1}, \ldots, Q_{N+1}$ are located in (weakly) general position. Denote by $H_{1}, \ldots, H_{N+1}$ the corresponding moving hypersurfaces.

By Lemma 3.1 and condition (iii), after passing to a subsequence, we may assume that for every $1 \leq k \leq N+1$, the divisors

$$
\left\{\nu\left(f^{(p)}, H_{k}\left(f^{(p)}\right)\right)\right\}=\left(f^{(p)}\right)^{-1}\left(H_{k}\left(f^{(p)}\right)\right) \quad(p \geq 1)
$$

are convergent (in the sense of convergence of divisors in $D$ ).

By a standard diagonal argument, we may assume that $D=B(R)$ and that $\left\{f^{(p)}\right\}$ meromorphically converges on $B(R)-S$ to a meromorphic mapping $f: B(R)-S \rightarrow \mathbb{P}^{N}(\mathbb{C})$.

We prove that there exists $k_{0} \in\{1, \ldots, N+1\}$ such that $f(D-S) \not \subset H_{k_{0}}$; more precisely, we prove that for any representation $f=\left(f_{0}: \cdots: f_{N}\right)$ of $f: D-S \rightarrow \mathbb{P}^{N}(\mathbb{C})$ (admissible or not), we have that $Q_{k_{0}}\left(f_{0}, \ldots, f_{N}\right) \not \equiv 0$ : $E=\left\{z \in D: f_{0}(z)=f_{1}(z)=\cdots=f_{N}(z)=0\right\}$ is a proper analytic subset. Since $Q_{1}, \ldots, Q_{N+1}$ are located in (weakly) general position, there exists $z \in D$ such that the system of equations

$$
\left\{\begin{array}{l}
Q_{k}(z)\left(\omega_{0}, \ldots, \omega_{N}\right)=0 \\
1 \leq k \leq N+1
\end{array}\right.
$$

has only the trivial solution $\omega=(0, \ldots, 0)$ in $\mathbb{C}^{N+1}$. But since then the same is true for the generic point $z \in D$, it is true in particular for the generic point $z \in D-E$. So for such point $z$ there exists some $k \in\{1, \ldots, N+1\}$ such that $Q_{k}(z)\left(f_{0}(z), \ldots, f_{N}(z)\right) \neq 0$. In order to simplify notations, from now on we put

$$
\begin{array}{ll}
Q^{(p)}:=Q_{k_{0}}\left(f^{(p)}\right), & Q:=Q_{k_{0}}, \\
H^{(p)}:=H_{k_{0}}\left(f^{(p)}\right), & H:=H_{k_{0}}, \quad d:=d_{k_{0}} .
\end{array}
$$

Let $z_{1}$ be any point of $S$. By [15, Theorem 3.6], for any $r(0<r<\tilde{R}=R-$ $\left.\left\|z_{1}\right\|\right)$, we can choose holomorphic functions $h^{(p)} \not \equiv 0$ and $h \not \equiv 0$ on $B\left(z_{1}, r\right)$ such that $\nu\left(f^{(p)}, H^{(p)}\right)=\nu_{h^{(p)}}, \nu=\nu_{h}$ for the limit $\nu$ of $\left\{\nu\left(f^{(p)}, H^{(p)}\right)\right\}$, and $\left\{h^{(p)}\right\}$ converges uniformly on compact subsets of $B\left(z_{1}, r\right)$ to $h$. Moreover, each $f^{(p)}$ has an admissible representation on $B\left(z_{1}, r\right)$

$$
f^{(p)}=\left(f_{0}^{(p)}: f_{1}^{(p)}: \cdots: f_{N}^{(p)}\right)
$$

with suitable holomorphic functions $f_{i}^{(p)}(0 \leq i \leq N)$ on $B\left(z_{1}, r\right)$. 
Let $z$ be a point in $B\left(z_{1}, r\right)-(S \cup\{h=0\})$. Choose a simply connected relatively compact neighborhood $W_{z}$ of $z$ in $B\left(z_{1}, r\right)-(S \cup\{h=0\})$ such that there exists a sequence $\left\{u_{z}^{(p)}\right\}$ of nonvanishing holomorphic functions on $W_{z}$ such that $\left\{u_{z}^{(p)} f_{i}^{(p)}\right\} \rightarrow f_{i}^{z}(0 \leq i \leq N)$ on $W_{z}$ and $f=\left(f_{0}^{z}: f_{1}^{z}: \cdots\right.$ : $\left.f_{N}^{z}\right)$ on $W_{z}$. It may be assumed that $h^{(p)}(p \geq 1)$ has no zero on $W_{z}$. We have $Q^{(p)}\left(f_{0}^{(p)}, \ldots, f_{N}^{(p)}\right)=v^{(p)} h^{(p)}$, where $v^{(p)}$ is a nonvanishing holomorphic function on $B\left(z_{1}, r\right)$. This implies that $Q^{(p)}\left(u_{z}^{(p)} f_{0}^{(p)}, \ldots, u_{z}^{(p)} f_{N}^{(p)}\right) \neq 0$ on $W_{z}$, since $Q^{(p)}$ is a homogeneous polynomial, and we have

$$
Q^{(p)}\left(u_{z}^{(p)} f_{0}^{(p)}, \ldots, u_{z}^{(p)} f_{N}^{(p)}\right) \rightarrow Q\left(f_{0}^{z}, \ldots, f_{N}^{z}\right)
$$

on $W_{z}$ since $Q^{(p)}$ converge uniformly on compact subsets of $D$ to $Q$. Since $f(D-S) \not \subset H$, it implies that $Q\left(f_{0}^{z}, \ldots, f_{N}^{z}\right) \not \equiv 0$ on $W_{z}$, and hence $Q\left(f_{0}^{z}, \ldots\right.$, $\left.f_{N}^{z}\right) \neq 0$ on $W_{z}$.

We recall that the $Q^{(p)}, p \geq 1$, and $Q$ have common degree $d$. Since

$$
\begin{aligned}
& Q^{(p)}\left(u_{z}^{(p)} f_{0}^{(p)}, \ldots, u_{z}^{(p)} f_{N}^{(p)}\right) \text { tends to } Q\left(f_{0}^{z}, \ldots, f_{N}^{z}\right) \text { on } W_{z} \quad \text { and } \\
& Q^{(p)}\left(u_{z}^{(p)} f_{0}^{(p)}, \ldots, u_{z}^{(p)} f_{N}^{(p)}\right)=\left(u_{z}^{(p)}\right)^{d} \cdot v^{(p)} \cdot h^{(p)}
\end{aligned}
$$

it follows that $\left(u_{z}^{(p)}\right)^{d} \cdot v^{(p)} \cdot h^{(p)}$ tends to $Q\left(f_{0}^{z}, \ldots, f_{N}^{z}\right)$ on $W_{z}$. Since $v^{(p)} \neq 0$ on $B\left(z_{1}, r\right), v^{(p)}=\left(k^{(p)}\right)^{d}$, where $k^{(p)}$ is a nonvanishing holomorphic function on $B\left(z_{1}, r\right)$. We have

$$
\left(u_{z}^{(p)}\right)^{d} \cdot\left(k^{(p)}\right)^{d}=\left(u_{z}^{(p)} \cdot k^{(p)}\right)^{d} \rightarrow \frac{Q\left(f_{0}^{z}, \ldots, f_{N}^{z}\right)}{h} \text { on } W_{z} .
$$

Define $F^{z}$ such that

$$
\left(F^{z}\right)^{d}:=\frac{Q\left(f_{0}^{z}, \ldots, f_{N}^{z}\right)}{h} \text { on } W_{z}
$$

We can do this because $\left(Q\left(f_{0}^{z}, \ldots, f_{N}^{z}\right)\right) / h \neq 0$ on $W_{z}$. So $\left(u_{z}^{(p)} \cdot k^{(p)}\right)^{d} \rightarrow$ $\left(F^{z}\right)^{d}$ on $W_{z}$; hence, $\left(\left(u_{z}^{(p)} \cdot k^{(p)}\right) / F^{z}\right)^{d}$ tends to 1 on $W_{z}$. Therefore, there exist infinite (or empty) subsets $\left\{N_{j}^{z}\right\}_{j=0}^{d-1}$ of $\mathbb{N}$ such that $\mathbb{N}$ is a disjoint union of sets $N_{j}^{z}$ and

$$
\left\{\frac{u_{z}^{(p)} \cdot k^{(p)}}{F^{z}}\right\}_{p \in N_{j}^{z}} \rightarrow \theta_{j}=e^{i \cdot(2 \pi j / d)} \quad \text { for each } 0 \leq j \leq d-1
$$


This implies that $\left\{f_{i}^{(p)} / k^{(p)}\right\}_{p \in N_{j}^{z}} \rightarrow F_{i}^{z} / \theta_{j}$ on $W_{z}$, where $F_{i}^{z}=f_{i}^{z} / F^{z}$ on $W_{z}$.

Take $a \in B\left(z_{1}, r\right)-(S \cup\{h=0\})$. Then $\left\{f_{i}^{(p)} / k^{(p)}\right\}_{p \in N_{j}^{a}} \rightarrow F_{i}^{a} / \theta_{j}$ on $W_{a}$ for each $0 \leq j \leq d-1$.

Take $b \in B\left(z_{1}, r\right)-(S \cup\{h=0\})$ such that $W_{a} \cap W_{b} \neq \emptyset$. We will prove that $\left\{f_{i}^{(p)} / k^{(p)}\right\}_{p \in N_{j}^{a}} \rightarrow F_{i}^{b} / \theta_{j} \cdot c$ for each $0 \leq j \leq d-1$. Indeed, without loss of generality, we may assume that $f_{0}^{a} \not \equiv 0$ on $W_{a}$. Then $f_{0}^{x} \not \equiv 0$ on $W_{x}$ for each $x \in B\left(z_{1}, r\right)-(S \cup\{h=0\})$. Hence, $F_{0}^{x} \not \equiv 0$ on $W_{x}$ for each $x \in B\left(z_{1}, r\right)-(S \cup\{h=0\})$.

Consider $\left|N_{j}^{a}\right|=\infty$, where $|\cdot|$ denotes the cardinality of a set.

Assume that there exist $N_{1}^{b}, N_{2}^{b}$ such that for $\tilde{N}:=N_{j}^{a} \cap N_{1}^{b}$ and $\tilde{\tilde{N}}:=$ $N_{j}^{a} \cap N_{2}^{b}$ we have $|\tilde{N}|=|\tilde{\tilde{N}}|=\infty$. Since $\left\{f_{0}^{(p)} / k^{(p)}\right\}_{p \in \tilde{N} \subset N_{1}^{b}} \rightarrow F_{0}^{b} / \theta_{1}$ on $W_{b}$ and $\left\{f_{0}^{(p)} / k^{(p)}\right\}_{p \in \tilde{N} \subset N_{j}^{a}} \rightarrow F_{0}^{a} / \theta_{j}$ on $W_{a}$, we have $F_{0}^{b} / \theta_{1}=F_{0}^{a} / \theta_{j}$ on $W_{a} \cap$ $W_{b}$. Similarly, $F_{0}^{b} / \theta_{2}=F_{0}^{a} / \theta_{j}$ on $W_{a} \cap W_{b}$. This is a contradiction. Thus, every infinite subset $N_{j}^{a}$ intersects and intersects infinitely only with the subset $N_{\alpha(j)}^{b}$. Moreover, $\left|N_{j}^{a} \Delta N_{\alpha(j)}^{b}\right|<\infty$, where $A \Delta B=(A-B) \cup(B-A)$ for arbitrary sets $A, B$.

From this it follows that there exists a bijection $\alpha:\{0,1, \ldots, d-1\} \rightarrow$ $\{0,1, \ldots, d-1\}$ such that

$$
\begin{aligned}
N_{j}^{a} & =\emptyset \quad \text { if and only if } N_{\alpha(j)}^{b}=\emptyset ; \\
\text { if }\left|N_{j}^{a}\right| & =\infty \quad \text { then }\left|N_{j}^{a} \Delta N_{\alpha(j)}^{b}\right|<\infty .
\end{aligned}
$$

On the other hand, since $\left\{f_{0}^{(p)} / k^{(p)}\right\}_{p \in N_{j}^{a} \cap N_{\alpha(j)}^{b}} \rightarrow F_{0}^{a} / \theta_{j}$ on $W_{a}$ and $\left\{f_{0}^{(p)} / k^{(p)}\right\}_{p \in N_{j}^{a} \cap N_{\alpha(j)}^{b}} \rightarrow F_{0}^{b} / \theta_{\alpha(j)}$ on $W_{b}$, we have $F_{0}^{a} / \theta_{j}=F_{0}^{b} / \theta_{\alpha(j)}$ on $W_{a} \cap$ $W_{b}$. This means that $F_{0}^{a}=F_{0}^{b} \cdot \theta_{j} / \theta_{\alpha(j)}$ on $W_{a} \cap W_{b}$ for each $0 \leq j \leq d-1$, and hence $c_{b}:=\theta_{j} / \theta_{\alpha(j)}$ is a constant independent of $j, 0 \leq j \leq d-1$. This implies that $\left\{f_{i}^{(p)} / k^{(p)}\right\}_{p \in N_{j}^{a} \cap N_{\alpha(j)}^{b}} \rightarrow F_{i}^{b} / \theta_{\alpha(j)}=F_{i}^{b} / \theta_{j} \cdot c_{b}$ on $W_{b}$, and hence

$$
\left\{\frac{f_{i}^{(p)}}{k^{(p)}}\right\}_{p \in N_{j}^{a}} \rightarrow \frac{F_{i}^{b}}{\theta_{j}} \cdot c_{b} \text { on } W_{b} .
$$

Applying this procedure a finite number of times, we have

$$
\left\{\frac{f_{i}^{(p)}}{k^{(p)}}\right\}_{p \in N_{j}^{a}} \rightarrow \frac{F_{i}^{x}}{\theta_{j}} \cdot c_{x}
$$


on $W_{x}$ for each $x \in B\left(z_{1}, r\right)-(S \cup\{h=0\})$ and for each $0 \leq j \leq d-1$. Indeed, by the assumption on the Hausdorff dimension of $S$ and by Corollary 2.2, the open set $B\left(z_{1}, r\right)-(S \cup\{h=0\})$ is pathwise connected, and such a path between $a$ and $x$, which is compact as the image of a closed interval under a continuous map, can be covered by a finite number of such neighborhoods $W_{y}$ with $y \in B\left(z_{1}, r\right)-(S \cup\{h=0\})$. And since the limit is unique if it exists, it does not depend on the choice of the path. For $p \in N_{j}^{a}$, put $\tilde{f}_{i}^{(p)}=f_{i}^{(p)} \cdot\left(\theta_{j} / k^{(p)}\right)(0 \leq i \leq N)$. Then $f^{(p)}=\left(\tilde{f}_{0}^{(p)}, \ldots, \tilde{f}_{N}^{(p)}\right)$ for all $p \in N_{j}^{a}$ and $0 \leq j \leq d-1$, and $\left\{\tilde{f}_{i}^{(p)}\right\}_{p=1}^{\infty} \rightarrow F_{i}^{x} \cdot c_{x}$ on $W_{x}$ for each $0 \leq i \leq N$. Note that if $W_{x} \cap W_{y} \neq \emptyset\left(x, y \in B\left(z_{1}, r\right)-(S \cup\{h=0\})\right)$, then $F_{i}^{x} \cdot c_{x}=F_{i}^{y} \cdot c_{y}$ for each $0 \leq i \leq N$.

Define the function $F_{i}: B\left(z_{1}, r\right)-(S \cup\{h=0\}) \rightarrow \mathbb{C}$ given by $\left.F_{i}\right|_{W_{z}}=$ $F_{i}^{z} \cdot c_{z}$. Then $\left\{\tilde{f}_{i}^{(p)}\right\}_{p=1}^{\infty} \rightarrow F_{i}$ on $B\left(z_{1}, r\right)-(S \cup\{h=0\})$ for each $0 \leq i \leq N$.

We now prove that the sequence $\left\{f^{(p)}\right\}_{p=1}^{\infty}$ meromorphically converges on $B\left(z_{1}, r\right)$ to some meromorphic mapping $\tilde{F}=\left(\tilde{F}_{0}, \ldots, \tilde{F}_{N}\right)$. Indeed, let $z^{(0)}$ be any point of $S_{1}=S \cup\{h=0\}$. Since $\Lambda^{2 n-1}\left(S_{1}\right)=0$, by Lemma 2.1(a) there exists a complex line $l_{z^{(0)}}$ passing through $z^{(0)}$ such that $\Lambda^{1}\left(S_{1} \cap l_{z^{(0)}}\right)=0$. Put $l_{z^{(0)}}=\left\{z^{(0)}+z \cdot u: z \in \mathbb{C}\right\}$. Then by Lemma 2.1(b) there exists $R>0$ such that

$$
\mathcal{C}_{0}=\left\{z^{(0)}+R \cdot e^{i \theta} \cdot u: \theta \in[0,2 \pi]\right\},
$$

satisfying $\mathcal{C}_{0} \subset B\left(z_{1}, r\right)$ and $\mathcal{C}_{0} \cap S_{1}=\emptyset$. By the maximum principle, it implies that the sequence $\left\{\tilde{f}_{i}^{(p)}\left(z^{(0)}\right)\right\}$ converges. Put $\lim _{p \rightarrow \infty} \tilde{f}_{i}^{(p)}\left(z^{(0)}\right)=$ $\tilde{F}_{i}\left(z^{(0)}\right)$. This means that the mapping $F_{i}$ extends over $B\left(z_{1}, r\right)$ to the mapping $\tilde{F}_{i}$.

We now prove that the sequence $\left\{\tilde{f}_{i}^{(p)}(z)\right\}_{p=1}^{\infty}$ converges uniformly on compact subsets of $B\left(z_{1}, r\right)$ to $\tilde{F}_{i}(z)$. Indeed, assume that $\left\{z^{(j)}\right\} \subset B\left(z_{1}, r\right)$ converges to $z^{(0)} \in B\left(z_{1}, r\right)$. As above, there exists a circle $\mathcal{C}_{0}=\left\{z^{(0)}+R\right.$. $\left.e^{i \theta} \cdot u: \theta \in[0,2 \pi]\right\} \subset B\left(z_{1}, r\right)$ such that $\mathcal{C}_{0} \cap S_{1}=\emptyset$. Since $\mathcal{C}_{0}$ is a compact subset of $B\left(z_{1}, r\right)-S_{1}$, there exists $\epsilon_{0}>0$ such that

$$
V\left(\mathcal{C}_{0}, \epsilon_{0}\right)=\left\{z \in \mathbb{C}^{n}: \operatorname{dist}\left(z, \mathcal{C}_{0}\right)<\epsilon_{0}\right\} \Subset B\left(z_{1}, r\right)-S_{1} .
$$

Consider the circles $\mathcal{C}_{j}=\left\{z^{(j)}+R \cdot e^{i \theta} \cdot u: \theta \in[0,2 \pi]\right\}$. It is easy to see that $\operatorname{dist}\left(\mathcal{C}_{0}, \mathcal{C}_{j}\right)=\left\|z^{(j)}-z^{(0)}\right\| \rightarrow 0$ as $j \rightarrow \infty$. Thus, without loss of generality, we may assume that $\mathcal{C}_{j} \subset V\left(\mathcal{C}_{0}, \epsilon_{0}\right) \Subset B\left(z_{1}, r\right)-S_{1}$. By the hypothesis, $\forall \epsilon>0$, $\exists N=N(\epsilon)$ such that

$$
\sup \left\{\left\|\tilde{f}_{i}^{(p)}(z)-F_{i}(z)\right\|: z \in V\left(\mathcal{C}_{0}, \epsilon_{0}\right), p \geq N\right\}<\epsilon .
$$


By the maximum principle, we have $\limsup _{j \rightarrow \infty}\left\|\tilde{f}_{i}^{(j)}\left(z^{(j)}\right)-F_{i}\left(z^{(j)}\right)\right\|=0$. This implies that the sequence $\left\{\tilde{f}_{i}^{(p)}\right\}_{p=1}^{\infty}$ converges uniformly on compact subsets of $B\left(z_{1}, r\right)$ to $\tilde{F}_{i}$. This finishes the proof of part (a) of the lemma.

In order to prove part (b), we first remark that it suffices to prove that $\left\{f^{(p)}\right\}$ has a subsequence which converges locally uniformly on $D$ to a holomorphic mapping $f$ of $D$ to $\mathbb{P}^{N}(\mathbb{C})$, which means that after passing to a subsequence we have the following.

Let $z_{1}$ be any point of $D$. Then there exists $r>0$ and, for each $f^{(p)}$, a holomorphic representation on $B\left(z_{1}, r\right)$

$$
f^{(p)}=\left(f_{0}^{(p)}: f_{1}^{(p)}: \cdots: f_{N}^{(p)}\right)
$$

with suitable holomorphic functions $f_{i}^{(p)}(0 \leq i \leq N)$ without common zeros on $B\left(z_{1}, r\right)$, such that $\left\{f_{i}^{(p)}\right\} \rightarrow f_{i}(0 \leq i \leq N)$ uniformly on $B\left(z_{1}, r\right)$ and $f=\left(f_{0}: f_{1}: \cdots: f_{N}\right)$ is a holomorphic map on $B\left(z_{1}, r\right)$; this means that the $f_{i}(0 \leq i \leq N)$ are without common zeros on $B\left(z_{1}, r\right)$.

By part (a) we know that $\left\{f^{(p)}\right\}$ has a subsequence which converges meromorphically on $D$ to a meromorphic mapping $f$ of $D$ to $\mathbb{P}^{N}(\mathbb{C})$, which means that after passing to a subsequence we have the following.

Let $z_{1}$ be any point of $D$. Then there exists $r>0$ and, for each $f^{(p)}$, an admissible representation on $B\left(z_{1}, r\right)$

$$
f^{(p)}=\left(f_{0}^{(p)}: f_{1}^{(p)}: \cdots: f_{N}^{(p)}\right)
$$

with suitable holomorphic functions $f_{i}^{(p)}(0 \leq i \leq N)$ on $B\left(z_{1}, r\right)$, such that $\left\{f_{i}^{(p)}\right\} \rightarrow f_{i}(0 \leq i \leq N)$ uniformly on $B\left(z_{1}, r\right)$ and $f=\left(f_{0}: f_{1}: \cdots: f_{N}\right)$ is a meromorphic map on $B\left(z_{1}, r\right)$. Observing that the admissible representations of the holomorphic maps $f^{(p)}=\left(f_{0}^{(p)}: f_{1}^{(p)}: \cdots: f_{N}^{(p)}\right)$ are automatically without common zeros, the only thing which remains to be proved is that under the conditions of (b) we have

$$
E=\left\{z \in B\left(z_{1}, r\right): f_{0}(z)=f_{1}(z)=\cdots=f_{N}(z)=0\right\}=\emptyset .
$$

We also recall that by the proof of part (a), we have that there exists $k_{0} \in\{1, \ldots, N+1\}$ such that $Q^{(p)}=Q_{k_{0}}\left(f^{(p)}\right), p \geq 1$, converge uniformly on compact subsets of $D$ to $Q=Q_{k_{0}}$, and $f(D-S) \not \subset H_{k_{0}}$; more precisely, we have that for any representation $f=\left(f_{0}: \cdots: f_{N}\right)$ of the meromorphic map $f: D \rightarrow \mathbb{P}^{N}(\mathbb{C})$ (admissible or not) we have

$$
Q\left(f_{0}, \ldots, f_{N}\right) \not \equiv 0
$$


Now we can end the proof with an easy application of Hurwitz's theorem, as follows. By the condition of (b) we have that, for all $p \geq 1$,

$$
Q^{(p)}\left(f_{0}^{(p)}, \ldots, f_{N}^{(p)}\right) \neq 0
$$

on $B\left(z_{1}, r\right)$. And we also have that

$$
Q^{(p)}\left(f_{0}^{(p)}, \ldots, f_{N}^{(p)}\right) \rightarrow Q\left(f_{0}, \ldots, f_{N}\right)
$$

uniformly on compact subsets of $B\left(z_{1}, r\right)$. By (3.3) and Hurwitz's theorem we get that $Q\left(f_{0}, \ldots, f_{N}\right) \neq 0$ on $B\left(z_{1}, r\right)$. But since $Q$ is a homogeneous polynomial, this implies that

$$
E=\left\{z \in B\left(z_{1}, r\right): f_{0}(z)=f_{1}(z)=\cdots=f_{N}(z)=0\right\}=\emptyset .
$$

We remark that the following corollary of Lemma 3.7(a) generalizes [2, Proposition 3.5].

COROllary 3.8. Let $\left\{f^{(p)}\right\}$ be a sequence of meromorphic mappings of a domain $D$ in $\mathbb{C}^{n}$ into $\mathbb{P}^{N}(\mathbb{C})$, and let $S$ be a closed subset of $D$ with $\Lambda^{2 n-1}(S)=0$. Suppose that $\left\{f^{(p)}\right\}$ meromorphically converges on $D-S$ to a meromorphic mapping $f$ of $D-S$ into $\mathbb{P}^{N}(\mathbb{C})$. If there exists a moving hypersurface $H$ in $\mathbb{P}^{N}(\mathbb{C})$ such that $f(D-S) \not \subset H$ and $\left\{\nu\left(f^{(p)}, H\right)\right\}$ is a convergent sequence of divisors on $D$, then $\left\{f^{(p)}\right\}$ is meromorphically convergent on $D$.

Lemma 3.9 ([19, Theorem 2.5]). Let $\mathcal{F}$ be a family of holomorphic mappings of a domain $D$ in $\mathbb{C}^{n}$ onto $\mathbb{P}^{N}(\mathbb{C})$. Then the family $\mathcal{F}$ is not normal on $D$ if and only if there exist a compact subset $K_{0} \subset D$ and sequences $\left\{f_{i}\right\} \subset \mathcal{F},\left\{z_{i}\right\} \subset K_{0},\left\{r_{i}\right\} \subset \mathbb{R}$ with $r_{i}>0$ and $r_{i} \longrightarrow 0^{+}$, and $\left\{u_{i}\right\} \subset \mathbb{C}^{n}$ which are unit vectors such that

$$
g_{i}(\xi):=f_{i}\left(z_{i}+r_{i} u_{i} \xi\right)
$$

where $\xi \in \mathbb{C}$ such that $z_{i}+r_{i} u_{i} \xi \in D$, converges uniformly on compact subsets of $\mathbb{C}$ to a nonconstant holomorphic map g of $\mathbb{C}$ to $\mathbb{P}^{N}(\mathbb{C})$.

Lemma $3.10\left(\left[9\right.\right.$, Theorem $\left.\left.4^{\prime}\right]\right)$. Suppose that $q \geq 2 N+1$ hyperplanes $H_{1}, \ldots, H_{q}$ are given in general position in $\mathbb{P}^{N}(\mathbb{C})$ and that $q$ positive integers (may be $\infty) m_{1}, \ldots, m_{q}$ are given such that

$$
\sum_{i=1}^{q}\left(1-\frac{N}{m_{j}}\right)>N+1 \text {. }
$$


Then there does not exist a nonconstant holomorphic mapping $f: \mathbb{C} \longrightarrow$ $\mathbb{P}^{N}(\mathbb{C})$ such that $f$ intersects $H_{j}$ with multiplicity at least $m_{j}(1 \leq j \leq q)$.

Lemma 3.11. Let $P_{0}, \ldots, P_{N}$ be $N+1$ homogeneous polynomials of common degree in $\mathbb{C}\left[x_{0}, \ldots, x_{n}\right]$. Also, let $\left\{Q_{j}\right\}_{j=1}^{q}(q \geq N+1)$ be homogeneous polynomials in $\mathcal{S}\left(\left\{P_{i}\right\}_{i=0}^{N}\right)$ such that

$$
D\left(Q_{1}, \ldots, Q_{q}\right)=\prod_{1 \leq j_{0}<\cdots<j_{N} \leq q} \inf _{\|\omega\|=1}\left(\left|Q_{j_{0}}(\omega)\right|^{2}+\cdots+\left|Q_{j_{N}}(\omega)\right|^{2}\right)>0,
$$

where $Q_{j}(\omega)=\sum_{|I|=d_{j}} a_{j I} \cdot \omega^{I}$.

Then $\left\{Q_{j}\right\}_{j=1}^{q}$ are located in general position in $\mathcal{S}\left(\left\{P_{i}\right\}_{i=0}^{N}\right)$, and $\left\{P_{i}\right\}_{i=0}^{N}$ are located in general position in $P^{N}(\mathbb{C})$ (see Sections 2.3 and 2.6).

Proof. (a) Suppose that $\left\{Q_{j}\right\}_{j=1}^{q}$ are not located in general position in $S\left(\left\{P_{i}\right\}_{i=0}^{N}\right)$. Then there exist $N+1$ polynomials in $\left\{Q_{j}\right\}_{j=1}^{q}$ which are not linearly independent. Without loss of generality, we may assume that

$$
Q_{N+1}=\sum_{j=1}^{N} b_{j} Q_{j} \quad\left(b_{j} \in \mathbb{C}\right)
$$

Then

$$
\begin{aligned}
X & =\left\{\omega \in \mathbb{C}^{N+1} \mid Q_{1}(\omega)=\cdots=Q_{N}(\omega)=Q_{N+1}(\omega)=0\right\} \\
& =\left\{\omega \in \mathbb{C}^{N+1} \mid Q_{1}(\omega)=\cdots=Q_{N}(\omega)=0\right\}
\end{aligned}
$$

is an analytic subset in $\mathbb{C}^{N+1}$. Since $\operatorname{dim} X \geq 1$, there exists $\omega_{0} \neq 0$ in $\mathbb{C}^{N+1}$ such that

$$
Q_{1}\left(\omega_{0}\right)=\cdots=Q_{N}\left(\omega_{0}\right)=Q_{N+1}\left(\omega_{0}\right)=0 .
$$

Moreover, since $\left\{Q_{j}\right\}_{j=1}^{q}$ are all homogeneous polynomials, we may assume that $\left\|\omega_{0}\right\|=1$. Thus, we have

$$
\left|Q_{1}\left(\omega_{0}\right)\right|^{2}+\cdots+\left|Q_{N+1}\left(\omega_{0}\right)\right|^{2}=0
$$

and hence

$$
D\left(Q_{1}, \ldots, Q_{q}\right)=0
$$

This is a contradiction. 
(b) Suppose that $\left\{P_{i}\right\}_{i=0}^{N}$ are not located in general position in $P^{N}(\mathbb{C})$. Then there exists $\omega_{0} \neq 0$ in $\mathbb{C}^{N+1}$ such that

$$
P_{0}\left(\omega_{0}\right)=\cdots=P_{N}\left(\omega_{0}\right)=0 .
$$

Therefore, we have $Q_{j}\left(\omega_{0}\right)=0$ for any $1 \leq j \leq q$, and thus again,

$$
D\left(Q_{1}, \ldots, Q_{q}\right)=0 .
$$

This is a contradiction.

Lemma 3.12. Let $f=\left(f_{0}: \cdots: f_{N}\right): \mathbb{C} \longrightarrow \mathbb{P}^{N}(\mathbb{C})$ be a holomorphic mapping, and let $\left\{P_{i}\right\}_{i=0}^{N}$ be $N+1$ homogeneous polynomials in general position of common degree in $\mathbb{C}\left[\omega_{0}, \ldots, \omega_{N}\right]$. Assume that

$$
F=\left(F_{0}: \cdots: F_{n}\right): \mathbb{P}^{N}(\mathbb{C}) \longrightarrow \mathbb{P}^{N}(\mathbb{C})
$$

is the mapping defined by

$$
F_{i}(\omega)=P_{i}(\omega) \quad(0 \leq i \leq N)
$$

Then, $F(f)$ is a constant map if and only if $f$ is a constant map.

Proof. Since $\left\{P_{i}\right\}_{i=0}^{N}$ are $N+1$ homogeneous polynomials in general position of common degree in $\mathbb{C}\left[\omega_{0}, \ldots, \omega_{N}\right], F$ is a morphism. Suppose that $F(f)=a$, where $a=\left(a_{0}: \cdots: a_{n}\right) \in \mathbb{P}^{N}(\mathbb{C})$. We have $f(\mathbb{C}) \subset F^{-1}(a)$. Suppose that $\operatorname{dim} F^{-1}(a) \geq 1$. Take $H$ any hyperplane in $\mathbb{P}^{N}(\mathbb{C})$ with $a \notin H$. Then $F^{-1}(H)$ is a hypersurface in $\mathbb{P}^{N}(\mathbb{C})$ since the $\left\{P_{i}\right\}_{i=0}^{N}$ are in general position, so in particular they are not linearly dependent. By Bézout's theorem there exists a point $\omega_{0} \in F^{-1}(a) \cap F^{-1}(H)$. Hence, $a=F\left(\omega_{0}\right) \in H$. This is a contradiction. Therefore, $\operatorname{dim} F^{-1}(a)=0$, so $F^{-1}(a)$ is a finite set. Since $f$ is continuous and $f(\mathbb{C}) \subset F^{-1}(a)$, it must be a constant map.

Lemma 3.13. Let $P_{0}, \ldots, P_{N}$ be $N+1$ homogeneous polynomials of common degree in $\mathbb{C}\left[\omega_{0}, \ldots, \omega_{N}\right]$, and let $\left\{Q_{j}\right\}_{j=1}^{q}(q \geq 2 N+1)$ be homogeneous polynomials in $\mathcal{S}\left(\left\{P_{i}\right\}_{i=0}^{N}\right)$ such that

$$
D\left(Q_{1}, \ldots, Q_{q}\right)>0 .
$$

Assume that $m_{1}, \ldots, m_{q}$ are positive integers (may be $\infty$ ) such that

$$
\sum_{j=1}^{q}\left(1-\frac{N}{m_{j}}\right)>N+1 .
$$


Then there does not exist a nonconstant holomorphic mapping

$$
f: \mathbb{C} \longrightarrow \mathbb{P}^{N}(\mathbb{C})
$$

such that $f$ intersects $Q_{j}$ with multiplicity at least $m_{j}(1 \leq j \leq q)$.

Proof. Suppose that $f: \mathbb{C} \longrightarrow \mathbb{P}^{N}(\mathbb{C})$ is a holomorphic mapping such that $f$ intersects $Q_{i}$ with multiplicity at least $m_{i}(1 \leq i \leq q)$. For each $1 \leq i \leq q$, we define

$$
Q_{j}=\sum_{i=0}^{N} b_{j i} P_{i}
$$

and

$$
H_{j}=\left\{\omega \in \mathbb{C}^{N+1} \mid \sum_{i=0}^{N} b_{j i} \omega_{i}=0\right\} .
$$

Let $\tilde{f}=\left(f_{0}, \ldots, f_{N}\right)$ be an admissible representation of $f$ on $\mathbb{C}$ (i.e., the $f_{0}, \ldots, f_{N}$ have no common zeros), and denote $F=\left(P_{0}(\widetilde{f}): \cdots: P_{N}(\widetilde{f})\right)$. By Lemma 3.11, $\left\{P_{i}\right\}_{i=0}^{N}$ are in general position in $\mathbb{P}^{N}(\mathbb{C})$, and $\left\{Q_{j}\right\}_{j=1}^{q}$ are located in general position in $\mathcal{S}\left(\left\{P_{i}\right\}_{i=0}^{N}\right)$. This means that the hyperplanes $\left\{H_{j}\right\}_{j=1}^{q}$ are located in general position in $\mathbb{P}^{N}(\mathbb{C})$. Since $f$ intersects $Q_{j}$ with multiplicity at least $m_{j}$ and

$$
Q_{j}(\tilde{f})=\left(\sum_{i=0}^{N} b_{j i} P_{i}\right)(\tilde{f})=\sum_{i=0}^{N} b_{j i}\left(P_{i}(\tilde{f})\right)
$$

$F$ also intersects $H_{j}$ with multiplicity at least $m_{j}(1 \leq j \leq q)$. By Lemma 3.10, $F$ is a constant map, and by Lemma 3.12, $f$ is a constant map, too.

LEMMA 3.14. Let natural numbers $N$ and $q \geq N+1$ be fixed. Let $T_{i}^{(p)}$ $(0 \leq i \leq N, p \geq 1)$ be moving hypersurfaces in $\mathbb{P}^{N}(\mathbb{C})$ of common degree $d^{(p)}$, and let $H_{j}^{(p)} \in \widetilde{\mathcal{S}}\left(\left\{T_{i}^{(p)}\right\}_{i=0}^{N}\right)(1 \leq j \leq q, p \geq 1)$ such that the following conditions are satisfied.

(i) For each $0 \leq i \leq N$, the coefficients of the homogeneous polynomials $P_{i}^{(p)}$ which define the $T_{i}^{(p)}$ are bounded above uniformly for all $p \geq 1$ on compact subsets of $D$, and for all $1 \leq j \leq q$, the coefficients $b_{i j}^{(p)}(z)$ of the linear combinations of the $P_{i}^{(p)}, i=0, \ldots, N$, which define the homogeneous polynomials $Q_{j}^{(p)}=\sum_{i=0}^{N} b_{i j}^{(p)} P_{i}^{(p)}$ which define the $H_{j}^{(p)}$ are bounded above uniformly for all $p \geq 1$ on compact subsets of $D$. 
(ii) There exists $z_{0} \in D$ such that

$$
\inf _{p \in \mathbb{N}}\left\{D\left(Q_{1}^{(p)}, \ldots, Q_{q}^{(p)}\right)\left(z_{0}\right)\right\}>0 .
$$

Then, we have the following.

(a) There exists a subsequence $\left\{j_{p}\right\} \subset \mathbb{N}$ such that for $0 \leq i \leq N, P_{i}^{\left(j_{p}\right)}$ converge uniformly on compact subsets of $D$ to not identically zero homogeneous polynomials $P_{i}$ (meaning that the $P_{i}^{\left(j_{p}\right)}$ and $P_{i}$ are homogeneous polynomials in $\widetilde{\mathcal{H}}_{D}\left[\omega_{0}, \ldots, \omega_{N}\right]$ of the same degree $d$, and all their coefficients converge uniformly on compact subsets of $D)$, and the $b_{i j}^{\left(j_{p}\right)}$ converge uniformly on compact subsets of $D$ to $b_{i j} \in \mathcal{H}_{D}$ for all $0 \leq i \leq N, 1 \leq j \leq q$.

(b) The $Q_{j}^{\left(j_{p}\right)}=\sum_{i=0}^{N} b_{i j}^{\left(j_{p}\right)} P_{i}^{\left(j_{p}\right)}$ converge, for all $0 \leq i \leq N, 1 \leq j \leq q$, uniformly on compact subsets of $D$ to $Q_{j}:=\sum_{i=0}^{N} b_{i j} P_{i} \in \widetilde{\mathcal{S}}\left(\left\{P_{i}\right\}_{i=0}^{N}\right)$, and we have

$$
D\left(Q_{1}, \ldots, Q_{q}\right)\left(z_{0}\right)>0 .
$$

In particular, the moving hypersurfaces $Q_{1}\left(z_{0}\right), \ldots, Q_{q}\left(z_{0}\right)$ are located in general position, and the moving hypersurfaces $Q_{1}(z), \ldots, Q_{q}(z)$ are located in (weakly) general position.

Proof. Since, by our conditions on the coefficients of the $P_{i}^{(p)}$ and on the $b_{i j}^{(p)}$, for all $1 \leq j \leq q$ the coefficients of the homogeneous polynomials $Q_{j}^{(p)}$ of degree $d^{(p)}$ are locally bounded uniformly for all $p \geq 1$ on compact subsets of $D$, all conditions of Lemma 3.6 are satisfied, and we get, after passing to a subsequence (which we denote for simplicity again by $\{p\} \subset \mathbb{N}$ ), that for $1 \leq j \leq q, Q_{j}^{(p)}$ converge uniformly on compact subsets of $D$ to not identically vanishing homogeneous polynomials $Q_{j}$ (meaning that the $Q_{j}^{(p)}$ and $Q_{j}$ are homogeneous polynomials in $\widetilde{\mathcal{H}}_{D}\left[\omega_{0}, \ldots, \omega_{N}\right]$ of the same degree $d_{j}$, and all their coefficients converge uniformly on compact subsets of $D$ ). Moreover (still by Lemma 3.6), we have that

$$
D\left(Q_{1}, \ldots, Q_{q}\right)\left(z_{0}\right)>0
$$

so the hypersurfaces $Q_{1}\left(z_{0}\right), \ldots, Q_{q}\left(z_{0}\right)$ are located in general position, and the moving hypersurfaces $Q_{1}(z), \ldots, Q_{q}(z)$ are located in (weakly) general position. Observe, moreover, that since all the $Q_{j}^{(p)}, 1 \leq j \leq q$, were of the same degree $d^{(p)}$, we have $d=d_{j}$ independent of $j$ for our subsequence. 
Hence, we have, for all $0 \leq i \leq N, p \geq 1$,

$$
P_{i}^{(p)}(z)(\omega)=\sum_{|I|=d} a_{i p I}(z) \cdot \omega^{I} .
$$

Now, since the $a_{j p I}(z)$ and the $b_{i j}^{(p)}(z)$ are locally bounded uniformly for all $p \geq 1$ on $D$, by using Montel's theorem and a standard diagonal argument with respect to an exhaustion of $D$ with compact subsets, after passing another time to a subsequence (which we denote for simplicity again by $\{p\} \subset \mathbb{N})$, we also can assume that $\left\{a_{i p I}(z)\right\}_{p=1}^{\infty}$ converges uniformly on compact subsets of $D$ to $a_{i I}$ for each $i, I$, and that $\left\{b_{i j}^{(p)}(z)\right\}_{p=1}^{\infty}$ converges uniformly on compact subsets of $D$ to $b_{i j}(z)$ for each $i, j$. Denote

$$
P_{i}(z)(\omega):=\sum_{|I|=d} a_{i I}(z) \cdot \omega^{I} .
$$

Since the limit is unique, then we have $Q_{j}=\sum_{i=0}^{N} b_{i j} P_{i}$ for $1 \leq j \leq q$, and in particular, we have that none of the $P_{0}(z), \ldots, P_{N}(z)$ is identically vanishing. (Otherwise, they could not be in (weakly) general position, which contradicted the general position of the $Q_{1}\left(z_{0}\right), \ldots, Q_{q}\left(z_{0}\right)$; in fact, if the $P_{i}\left(z_{0}\right)(\omega)$ had a nonzero solution $\omega_{0}$ in common, so would the $Q_{j}\left(z_{0}\right)(\omega)$.) Hence, $Q_{j} \in \widetilde{\mathcal{S}}\left(\left\{P_{i}\right\}_{i=0}^{N}\right)$, which completes the proof.

\section{§4. Proofs of the Theorems}

Proofs of Theorem 1.1 and Theorem 1.2. Let $\left\{f^{(p)}\right\}$ be a sequence of meromorphic mappings in $\mathcal{F}$. We have to prove that after passing to a subsequence (which we denote again by $\left\{f^{(p)}\right\}$ ), the sequence $\left\{f^{(p)}\right\}$ converges meromorphically on $D$ to a meromorphic mapping $f$. Moreover, under the stronger conditions of Theorem 1.2, we have to show that $\left\{f^{(p)}\right\}$ converges uniformly on compact subsets of $D$ to a holomorphic mapping $f$.

In order to simplify notation, we denote, for $1 \leq k \leq q$,

$$
Q_{k}^{(p)}:=Q_{k}\left(f^{(p)}\right) \quad \text { and } \quad H_{k}^{(p)}:=H_{k}\left(f^{(p)}\right) .
$$

By Lemma 3.6, after passing to a subsequence, for all $1 \leq k \leq q, Q_{k}^{(p)}$ converge uniformly on compact subsets of $D$ to $Q_{k}$, meaning that

$$
Q_{k}^{(p)}=Q_{k}^{(p)}(z)(\omega)=\sum_{|I|=d_{k}} a_{k p I}(z) \cdot \omega^{I}
$$


and

$$
Q_{k}=Q_{k}(z)(\omega)=\sum_{|I|=d_{k}} a_{k I}(z) \cdot \omega^{I}
$$

are homogeneous polynomials in $\widetilde{\mathcal{H}}_{D}\left[\omega_{0}, \ldots, \omega_{N}\right]$ of the same degree $d_{k}$, and that all their coefficients $a_{k p I}$ converge uniformly on compact subsets of $D$ to $a_{k I}$. Moreover, $Q_{1}, \ldots, Q_{q}$ are located in (weakly) general position.

By condition (ii) of Theorems 1.1 and 1.2 and Lemmas 3.1 and 3.2, and by condition (iii) of Theorems 1.1 and 1.2 and Lemmas 3.3 and 3.4, after passing to a subsequence, we may assume that the sequence $\left\{f^{(p)}\right\}$ satisfies

$$
\lim _{p \rightarrow \infty}\left(f^{(p)}\right)^{-1}\left(H_{k}^{(p)}\right)=S_{k} \quad(1 \leq k \leq N+1)
$$

as a sequence of closed subsets of $D$, where $S_{k}$ are either empty or pure $(n-1)$-dimensional analytic sets in $D$, and

$$
\lim _{p \rightarrow \infty}\left(f^{(p)}\right)^{-1}\left(H_{k}^{(p)}\right)-S=S_{k} \quad(N+2 \leq k \leq q)
$$

as a sequence of closed subsets of $D-S$, where $S_{k}$ are either empty or pure $(n-1)$-dimensional analytic sets in $D-S$.

Let $T=\left(\ldots, t_{k I}, \ldots\right)\left(1 \leq k \leq q,|I| \leq M:=\max \left\{d_{1}, \ldots, d_{q}\right\}\right)$ be a family of variables. Set $\widetilde{Q}_{k}=\sum_{|I| \leq M} t_{k I} \omega^{I} \in \mathbb{Z}[T, \omega](1 \leq k \leq q)$. For each subset $L \subset\{1, \ldots, q\}$ with $|L|=n+1$, take $\widetilde{R}_{L}$ is the resultant of the $\widetilde{Q}_{k}(k \in L)$. Since $\left\{Q_{k}\right\}_{k \in L}$ are in the (weakly) general position, $\widetilde{R}_{L}\left(\ldots, a_{k I}, \ldots\right) \not \equiv 0$ (where we put $a_{k I}=0$ for $|I| \neq d_{k}$ ). We set

$\widetilde{S}:=\left\{z \in D \mid \widetilde{R}_{L}\left(\ldots, a_{k I}, \ldots\right)=0\right.$ for some $L \subset\{1, \ldots, q\}$ with $\left.|L|=n+1\right\}$.

Let $E=\left(\bigcup_{k=1}^{q} S_{k} \cup \widetilde{S}\right)-S$. Then $E$ is either empty or a pure $(n-1)$ dimensional analytic set in $D-S$.

Fix any point $z_{1}$ in $(D-S)-E$. Choose a relatively compact neighborhood $U_{z_{1}}$ of $z_{1}$ in $(D-S)-E$. Then $\left\{\left.f^{(p)}\right|_{U_{z_{1}}}\right\} \subset \operatorname{Hol}\left(U_{z_{1}}, \mathbb{P}^{N}(\mathbb{C})\right)$. We now prove that the family $\left\{\left.f^{(p)}\right|_{U_{z_{1}}}\right\}$ is a holomorphically normal family. Indeed, suppose that the family $\left\{\left.f^{(p)}\right|_{U_{z_{1}}}\right\}$ is not holomorphically normal. By Lemma 3.9, there exist a subsequence (again denoted by $\left\{\left.f^{(p)}\right|_{U_{z_{1}}}\right\}_{p=1}^{\infty}$ ) and $P_{0} \in U_{z_{1}},\left\{P_{p}\right\}_{p=1}^{\infty} \subset U_{z_{1}}$ with $P_{p} \rightarrow P_{0},\left\{r_{p}\right\} \subset(0,+\infty)$ with $r_{p} \rightarrow 0^{+}$, and $\left\{u_{p}\right\} \subset \mathbb{C}^{n}$, which are unit vectors, such that $g_{p}(z):=f^{(p)}\left(P_{p}+r_{p} u_{p} z\right)$ 
converges uniformly on compact subsets of $\mathbb{C}$ to a nonconstant holomorphic map $g$ of $\mathbb{C}$ into $\mathbb{P}^{N}(\mathbb{C})$. Then, there exist admissible representations $g^{(p)}=\left(g_{0}^{(p)}: \cdots: g_{N}^{(p)}\right)$ of $g^{(p)}$ and an admissible representation $g=\left(g_{0}: \cdots:\right.$ $\left.g_{N}\right)$ of $g$ such that the $\left\{g_{i}^{(p)}\right\}$ converge uniformly on compact subsets of $\mathbb{C}$ to $g_{i}(0 \leq i \leq N)$. (Observe that an admissible representation of a holomorphic map is automatically without common zeros.) This implies that $Q_{k}^{(p)}\left(P_{p}+r_{p} u_{p} z\right)\left(g_{0}^{(p)}(z), \ldots, g_{n}^{(p)}(z)\right)$ converges uniformly on compact subsets of $\mathbb{C}$ to $Q_{k}\left(P_{0}\right)\left(g_{0}(z), \ldots, g_{N}(z)\right),(1 \leq k \leq q)$. Thus, by Hurwitz's theorem, one of the following two assertions holds:

(i) $Q_{k}\left(P_{0}\right)\left(g_{0}(z), \ldots, g_{N}(z)\right)=0$ on $\mathbb{C}$, that is, $g(\mathbb{C}) \subset H_{k}\left(P_{0}\right)$;

(ii) $Q_{k}\left(P_{0}\right)\left(g_{0}(z), \ldots, g_{N}(z)\right) \neq 0$ on $\mathbb{C}$, that is, $g(\mathbb{C}) \cap H_{k}\left(P_{0}\right)=\emptyset$.

Denote by $J$ the set of all indices $k \in\{1, \ldots, q\}$ with $g(\mathbb{C}) \subset H_{k}\left(P_{0}\right)$. Set $X=\bigcap_{k \in J} H_{k}\left(P_{0}\right)$ if $J \neq \emptyset$ and $X=\mathbb{P}^{N}(\mathbb{C})$ if $J=\emptyset$. Since $\mathbb{C}$ is irreducible, there exists an irreducible component $Z$ of $X$ such that $g(\mathbb{C}) \subset$ $Z-\left(\bigcup_{k \notin J} H_{k}\left(P_{0}\right)\right)$. Since $P_{0} \in U_{z_{1}}$, it implies that $\left\{H_{k}\left(P_{0}\right)\right\}_{k=1}^{q}$ are in general position in $\mathbb{P}^{N}(\mathbb{C})$, meaning that

$$
\text { for all } I \subset\{1, \ldots, q\} \text { with } \# I=N+1, \bigcap_{k \in I} H_{k}\left(P_{0}\right)=\emptyset \text {. }
$$

Put $J^{c}:=\{1, \ldots, q\} \backslash J$, and put $m:=\operatorname{dim}_{\mathbb{C}} Z$. We claim that for the hypersurfaces $\left\{H_{k}\left(P_{0}\right)\right\}_{k \in J^{c}}$ in $\mathbb{P}^{N}(\mathbb{C})$ we have

$$
\# J^{c} \geq 2 m+1 ; \text { for all } I \subset J^{c} \text { with } \# I=m+1, Z \cap\left(\bigcap_{k \in I} H_{k}\left(P_{0}\right)\right)=\emptyset \text {. }
$$

In fact, if $J=\emptyset$, so $X=\mathbb{P}^{N}(\mathbb{C})$, this holds since $q \geq 2 N+1$ and by (4.1). If $J \neq \emptyset$, the key observation is that by (4.1) and by Bézout's theorem we have

for all $1 \leq l \leq q$, for all $I \subset\{1, \ldots, q\}$ with $\# I=l$,

$$
\bigcap_{k \in I} H_{k}\left(P_{0}\right) \text { is of pure dimension } \operatorname{dim}_{\mathbb{C}} \bigcap_{k \in I} H_{k}\left(P_{0}\right)=\max \{N-l,-1\}
$$

(in particular, all irreducible components of $\bigcap_{k \in I} H_{k}\left(P_{0}\right)$ are of the same dimension), where $\operatorname{dim}_{\mathbb{C}}(\emptyset)=-1$. From that we first get

$$
m=\operatorname{dim}_{\mathbb{C}} Z=\operatorname{dim}_{\mathbb{C}} \bigcap_{k \in J} H_{k}\left(P_{0}\right)=\max \{N-\# J,-1\} .
$$


Since $g(\mathbb{C}) \subset Z$, so $m \geq 0$, we get

$$
\# J=N-m \text {. }
$$

Hence,

$$
\# J^{c}=q-\# J \geq(2 N+1)-(N-m)=N+m+1 \geq 2 m+1 .
$$

Moreover, if $I \subset J^{c}$ with $\# I=m+1$, then by (4.4)

$$
\#(I \cup J)=(m+1)+(N-m)=N+1
$$

and

$$
Z \cap \bigcap_{k \in I} H_{k}\left(P_{0}\right) \subset \bigcap_{k \in J} H_{k}\left(P_{0}\right) \cap \bigcap_{k \in I} H_{k}\left(P_{0}\right)=\bigcap_{k \in I \cup J} H_{k}\left(P_{0}\right)=\emptyset,
$$

where the last equality follows from (4.1). This proves (4.2) in the case $J \neq \emptyset$. By (4.2) and by [3, Corollary 1] (or by the more general [10, Theorem 7.3.4]), we get that $Z-\left(\bigcup_{k \notin J} H_{k}\left(P_{0}\right)\right)$ is complete hyperbolic and hyperbolically embedded, and hence $g$ is constant. This is a contradiction.

Thus, $\left\{f^{(p)}\right\}$ is a holomorphically normal family on $U_{z_{1}}$. By the usual diagonal argument, we can find a subsequence (again denoted by $\left\{f^{(p)}\right\}$ ) which converges uniformly on compact subsets of $(D-S)-E$ to a holomorphic mapping $f$ of $(D-S)-E$ into $\mathbb{P}^{N}(\mathbb{C})$.

By Lemma 3.7(a), $\left\{f^{(p)}\right\}$ has a meromorphically convergent subsequence (again denoted by $\left\{f^{(p)}\right\}$ ) on $D-S$, and again by Lemma 3.7(a), \{f(p) $\}$ has a meromorphically convergent subsequence on $D$. Then $\mathcal{F}$ is a meromorphically normal family on $D$. The proof of Theorem 1.1 is completed.

Under the additional conditions of Theorem 1.2 by Lemma 3.7(b), $\left\{f^{(p)}\right\}$ has a subsequence which converges uniformly on compact subsets of $D$ to a holomorphic mapping of $D$ to $\mathbb{P}^{N}(\mathbb{C})$. The proof of Theorem 1.2 is completed.

Proof of Theorem 1.3. Suppose that $\mathcal{F}$ is not normal on $D$. Then, by Lemma 3.9, there exists a subsequence denoted by $\left\{f^{(p)}\right\} \subset \mathcal{F}$ and $z_{0} \in$ $D,\left\{z_{p}\right\}_{p=1}^{\infty} \subset D$ with $z_{p} \rightarrow z_{0},\left\{r_{p}\right\} \subset(0,+\infty)$ with $r_{p} \rightarrow 0^{+}$, and $\left\{u_{p}\right\} \subset$ $\mathbb{C}^{n}$, which are unit vectors, such that $g^{(p)}(\xi):=f^{(p)}\left(z_{p}+r_{p} u_{p} \xi\right)$ converges uniformly on compact subsets of $\mathbb{C}$ to a nonconstant holomorphic map $g$ of $\mathbb{C}$ into $\mathbb{P}^{N}(\mathbb{C})$. 
By condition (i) of Theorem 1.3 and by Lemma 3.14, there exists a subsequence (which we denote again by $\{p\} \subset \mathbb{N}$ ) such that for $0 \leq i \leq N$, $P_{i}^{(p)}:=P_{i}\left(f^{(p)}\right)$ converge uniformly on compact subsets of $D$ to $P_{i}$; that the $b_{i j}^{(p)}:=b_{i j}\left(f^{(p)}\right)$ converge uniformly on compact subsets of $D$ to $b_{i j}$ for all $0 \leq i \leq N, 1 \leq j \leq q$; that the $Q_{j}^{(p)}:=Q_{j}\left(f^{(p)}\right)=\sum_{i=0}^{N} b_{i j}^{(p)} P_{i}^{(p)}$ converge, for all $0 \leq i \leq N, 1 \leq j \leq q$, uniformly on compact subsets of $D$ to $Q_{j}:=\sum_{i=0}^{N} b_{i j} P_{i} \in \overline{\widetilde{\mathcal{S}}}\left(\left\{P_{i}\right\}_{i=0}^{N}\right)$; and that we have, for any fixed $z=z_{0} \in D$,

$$
D\left(Q_{1}, \ldots, Q_{q}\right)(z)>\delta(z)>0
$$

(in particular, the moving hypersurfaces $Q_{1}(z), \ldots, Q_{q}(z)$ are located in (pointwise) general position). We finally recall that with writing both variables $z \in D$ and $\omega \in \mathbb{P}^{N}(\mathbb{C})$, we thus have that

$$
P_{i}^{(p)}(z)(\omega) \rightarrow P_{i}(z)(\omega) ; \quad Q_{j}^{(p)}(z)(\omega) \rightarrow Q_{j}(z)(\omega) ; \quad b_{i j}^{(p)}(z) \rightarrow b_{i j}(z)
$$

uniformly on compact subsets in the variable $z \in D$.

For any fixed $\xi_{0} \in \mathbb{C}$, there exists a ball $B\left(\xi_{0}, r_{0}\right)$ in $\mathbb{C}$ and an index $i$ such that $g\left(B\left(\xi_{0}, r_{0}\right)\right) \subset\left\{\omega \in \mathbb{P}^{N}(\mathbb{C}): \omega_{i} \neq 0\right\}$. Without loss of generality, we may assume that $i=0$. Therefore, there exist admissible representations

$$
\begin{aligned}
\tilde{g}^{(p)}(\xi) & =\left(1, g_{1}^{(p)}(\xi), \ldots, g_{N}^{(p)}(\xi)\right), \\
\tilde{g}(\xi) & =\left(1, g_{1}(\xi), \ldots, g_{N}(\xi)\right)
\end{aligned}
$$

of $g^{(p)}$ and $g$ on $B\left(\xi_{0}, r_{0}\right)$.

Because of the convergence of $\left\{g^{(p)}\right\}$ on $B\left(\xi_{0}, r_{0}\right),\left\{g_{i}^{(p)}\right\}$ converges uniformly on compact subsets of $B\left(\xi_{0}, r_{0}\right)$ to $g_{i}$ for each $1 \leq i \leq N$. This implies that $Q_{j}^{(p)}\left(z_{p}+r_{p} u_{p} \xi\right)\left(\widetilde{g}^{(p)}(\xi)\right)$ converges uniformly on compact subsets of $\mathbb{C}$ to $Q_{j}\left(z_{0}\right)(\widetilde{g}(\xi))$ and that $P_{i}^{(p)}\left(z_{p}+r_{p} u_{p} \xi\right)\left(\widetilde{g}^{(p)}(\xi)\right)$ converges uniformly on compact subsets of $\mathbb{C}$ to $P_{i}\left(z_{0}\right)(\widetilde{g}(\xi))$.

By Hurwitz's theorem, there exists a positive integer $N_{0}$ such that $Q_{j}^{(p)}\left(z_{p}+r_{p} u_{p} \xi\right)\left(\widetilde{g}^{(p)}(\xi)\right)$ and $Q_{j}\left(z_{0}\right)(\widetilde{g}(\xi))$ have the same number of zeros with counting multiplicities on $B\left(\xi_{0}, r_{0}\right)$ for each $p \geq N_{0}$. Since the map $g^{(p)}$ of $B\left(\xi_{0}, r_{0}\right)$ into $\mathbb{P}^{N}(\mathbb{C})$ intersects $Q_{j}^{(p)}$ with multiplicity at least $m_{j}$, it implies that any zero $\xi$ of $Q_{j}\left(z_{0}\right)(\widetilde{g}(\xi))$ has multiplicity at least $m_{j}$. Hence, $g$ intersects $Q_{j}\left(z_{0}\right)$ with multiplicity at least $m_{j}$ for each $1 \leq j \leq q$.

Since we have that $Q_{1}, \ldots, Q_{q}$ are in $\widetilde{\mathcal{S}}\left(\left\{P_{i}\right\}_{i=0}^{N}\right)$ and that

$$
D\left(Q_{1}, \ldots, Q_{q}\right)(z)>0 \text { for any } z \in D
$$


we have in particular that $Q_{1}\left(z_{0}\right), \ldots, Q_{q}\left(z_{0}\right)$ are in $\mathcal{S}\left(\left\{P_{i}\left(z_{0}\right)\right\}_{i=0}^{N}\right)$ and that

$$
D\left(Q_{1}, \ldots, Q_{q}\right)\left(z_{0}\right)>0 .
$$

Thus, by Lemma $3.13, g$ is a constant mapping of $\mathbb{C}$ into $\mathbb{P}^{N}(\mathbb{C})$. This is a contradiction.

Proofs of Theorem 1.4 and Theorem 1.5. Let $\left\{f^{(p)}\right\}$ be a sequence of meromorphic mappings in $\mathcal{F}$. We have to prove that after passing to a subsequence (which we denote again by $\left\{f^{(p)}\right\}$ ), the sequence $\left\{f^{(p)}\right\}$ converges meromorphically on $D$ to a meromorphic mapping $f$. Moreover, under the stronger conditions of Theorem 1.5, we have to show that $\left\{f^{(p)}\right\}$ converges uniformly on compact subsets of $D$ to a holomorphic mapping $f$.

By condition (i) of the theorems and by Lemma 3.14, there exists a subsequence (which we denote again by $\left\{f^{(p)}\right\}$ ) such that for $0 \leq i \leq N, P_{i}^{(p)}:=$ $P_{i}\left(f^{(p)}\right)$ are homogeneous polynomials of the same degree $d$ and converge uniformly on compact subsets of $D$ to $P_{i}$; that the $b_{i j}^{(p)}:=b_{i j}\left(f^{(p)}\right)$ converge uniformly on compact subsets of $D$ to $b_{i j}$ for all $0 \leq i \leq N, 1 \leq j \leq q$; that the $Q_{j}^{(p)}:=Q_{j}\left(f^{(p)}\right)=\sum_{i=0}^{N} b_{i j}^{(p)} P_{i}^{(p)}$ converge, for all $0 \leq i \leq N, 1 \leq j \leq q$, uniformly on compact subsets of $D$ to $Q_{j}:=\sum_{i=0}^{N} b_{i j} P_{i} \in \widetilde{\mathcal{S}}\left(\left\{P_{i}\right\}_{i=0}^{N}\right)$; and that

$$
D\left(Q_{1}, \ldots, Q_{q}\right)\left(z_{0}\right)>0 .
$$

In particular, the moving hypersurfaces $Q_{1}\left(z_{0}\right), \ldots, Q_{q}\left(z_{0}\right)$ are located in general position, and the moving hypersurfaces $Q_{1}(z), \ldots, Q_{q}(z)$ are located in (weakly) general position.

By condition (ii) of Theorem 1.4 and Lemmas 3.1 and 3.2, and by condition (iii) of the theorems and Lemmas 3.3 and 3.4, after passing to a subsequence, we may assume that the sequence $\left\{f^{(p)}\right\}$ satisfies

$$
\lim _{p \rightarrow \infty}\left(f^{(p)}\right)^{-1}\left(H_{k}^{(p)}\right)=S_{k} \quad(1 \leq k \leq N+1)
$$

as a sequence of closed subsets of $D$, where $S_{k}$ are either empty or pure $(n-1)$-dimensional analytic sets in $D$, and satisfies

$$
\begin{aligned}
& \lim _{p \rightarrow \infty} \overline{\left\{z \in \operatorname{Supp} \nu\left(f^{(p)}, H_{k}^{(p)}\right) \mid \nu\left(f^{(p)}, H_{k}^{(p)}\right)(z)<m_{k}\right\}}-S=S_{k} \\
& \quad(N+2 \leq k \leq q)
\end{aligned}
$$

as a sequence of closed subsets of $D-S$, where $S_{k}$ are either empty or pure $(n-1)$-dimensional analytic sets in $D-S$. 
Let $T=\left(\ldots, t_{k I}, \ldots\right)(1 \leq k \leq q,|I|=d)$ be a family of variables. Set $\widetilde{Q}_{k}=\sum_{|I|=d} t_{k I} \omega^{I} \in \mathbb{Z}[T, \omega](1 \leq k \leq q)$. For each subset $L \subset\{1, \ldots, q\}$ with $|L|=n+1$, take $\widetilde{R}_{L}$ is the resultant of the $\widetilde{Q}_{k}(k \in L)$. Since $\left\{Q_{k}\right\}_{k \in L}$ are in (weakly) general position, $\widetilde{R}_{L}\left(\ldots, a_{k I}, \ldots\right) \not \equiv 0$ (where we put $a_{k I}=0$ for $|I| \neq d)$. We set

$\widetilde{S}:=\left\{z \in D \mid \widetilde{R}_{L}\left(\ldots, a_{k I}, \ldots\right)=0\right.$ for some $L \subset\{1, \ldots, q\}$ with $\left.|L|=n+1\right\}$.

Let $E=\left(\bigcup_{k=1}^{q} S_{k} \cup \widetilde{S}\right)-S$. Then $E$ is either empty or a pure $(n-1)$ dimensional analytic set in $D-S$.

Fix any point $z_{1}$ in $(D-S)-E$. Choose a relatively compact neighborhood $U_{z_{1}}$ of $z_{1}$ in $(D-S)-E$. Then $\left\{\left.f^{(p)}\right|_{U_{z_{1}}}\right\} \subset \operatorname{Hol}\left(U_{z_{1}}, \mathbb{P}^{N}(\mathbb{C})\right)$. We now prove that the family $\left\{\left.f^{(p)}\right|_{U_{z_{1}}}\right\}$ is a holomorphically normal family. For this it is sufficient to observe that the family $\left\{\left.f^{(p)}\right|_{U_{z_{1}}}\right\}$ now satisfies all conditions of Theorem 1.3. In fact, there exists $N_{0}$ such that, for $p \geq N_{0}$, $\left\{\left.f^{(p)}\right|_{U_{z_{1}}}\right\}$ does not intersect $H_{k}^{(p)}$ for $1 \leq k \leq N+1$ and $\left\{\left.f^{(p)}\right|_{U_{z_{1}}}\right\}$ intersects $H_{k}^{(p)}$ of order at least $m_{k}$ for $N+2 \leq k \leq q$; and for all $z \in U_{z_{1}}$, we have $D\left(Q_{1}, \ldots, Q_{q}\right)(z)>0$. So if we still put $m_{k}=\infty$ for $1 \leq k \leq N+1$, the conditions of Theorem 1.3 are satisfied, and so the family $\left\{\left.f^{(p)}\right|_{U_{z_{1}}}\right\}$ is a holomorphically normal family. By the usual diagonal argument, we can find a subsequence (again denoted by $\left\{f^{(p)}\right\}$ ) which converges uniformly on compact subsets of $(D-S)-E$ to a holomorphic mapping $f$ of $(D-S)-E$ into $\mathbb{P}^{N}(\mathbb{C})$.

By Lemma 3.7(a), $\left\{f^{(p)}\right\}$ has a meromorphically convergent subsequence (again denoted by $\left\{f^{(p)}\right\}$ ) on $D-S$, and again by Lemma 3.7(a), $\left\{f^{(p)}\right\}$ has a meromorphically convergent subsequence on $D$. Then $\mathcal{F}$ is a meromorphically normal family on $D$. The proof of Theorem 1.4 is completed.

Under the additional conditions of Theorem 1.5, by Lemma 3.7(b) $\left\{f^{(p)}\right\}$ has a subsequence which converges uniformly on compact subsets of $D$ to a holomorphic mapping of $D$ to $\mathbb{P}^{N}(\mathbb{C})$. The proof of Theorem 1.5 is completed.

Acknowledgments. The authors' work was partially supported by grant 101.04-2014.48 from the National Foundation for Science and Technology Development of Vietnam. This work was done during a visit to the Vietnam Institute for Advanced Study in Mathematics (VIASM), and we thank the staff there for their hospitality and, in particular, VIASM for partial support. 


\section{REFERENCES}

[1] G. Aladro and S. G. Krantz, A criterion for normality in $\mathbb{C}^{n}$, J. Math. Anal. Appl. 161 (1991), 1-8. MR 1127544. DOI 10.1016/0022-247X(91)90356-5.

[2] E. M. Chirka, Complex Analytic Sets, Math. Appl. (Soviet Ser.) 46, Kluwer, Dordrecht, 1989. MR 1111477. DOI 10.1007/978-94-009-2366-9.

[3] A. Eremenko, A Picard type theorem for holomorphic curves, Period. Math. Hungar. 38 (1999), 39-42. MR 1721476. DOI 10.1023/A:1004794914744.

[4] H. Fujimoto, On families of meromorphic maps into the complex projective space, Nagoya Math. J. 54 (1974), 21-51. MR 0367301.

[5] J. E. Joseph and M. H. Kwack, Some classical theorems and families of normal maps in several complex variables, Complex Var. Theory Appl. 29 (1996), 343-362. MR 1390619.

[6] - Extension and convergence theorems for families of normal maps in several complex variables, Proc. Amer. Math. Soc. 125 (1997), 1675-1684. MR 1423310. DOI 10.1090/S0002-9939-97-04117-8.

[7] O. Lehto and K. I. Virtanen, Boundary behaviour and normal meromorphic functions, Acta Math. 97 (1957), 47-65. MR 0087746.

[8] P. N. Mai, D. D. Thai, and P. N. T. Trang, Normal families of meromorphic mappings of several complex variables into $\mathbb{P}^{N}(\mathbb{C})$, Nagoya Math. J. 180 (2005), 91-110. MR 2186670.

[9] E. I. Nochka, On the theory of meromorphic functions (in Russian), Dokl. Akad. Nauk SSSR 269, no. 3 (1983), 547-552; English translation in Soviet Math. Dokl. 27, no. 2 (1983), 377-381. MR 0701289.

[10] J. Noguchi and J. Winkelmann, Nevanlinna Theory in Several Complex Variables and Diophantine Approximation, Grundlehren Math. Wiss. 350, Springer, Tokyo, 2014. MR 3156076.

[11] S. D. Quang and T. V. Tan, Normal families of meromorphic mappings of several complex variables into $\mathbb{C} P^{N}$ for moving hypersurfaces, Ann. Polon. Math. 94 (2008), 97-110. MR 2438852. DOI 10.4064/ap94-2-1.

[12] M. Ru and W. Stoll, "The Cartan conjecture for moving targets" in Several Complex Variables and Complex Geometry, Part 2 (Santa Cruz, Calif., 1989), Proc. Sympos. Pure Math. 52, Amer. Math. Soc., Providence, 1991, 477-508. MR 1128565.

[13] — The second main theorem for moving targets, J. Geom. Anal. 1 (1991), 99138. MR 1113373. DOI 10.1007/BF02938116.

[14] H. Rutishauser, Über Folgen und Scharen von analytischen und meromorphen Funktionen mehrerer Variabeln, sowie von analytischen Abbildungen, Acta Math. 83 (1950), 249-325. MR 0036322.

[15] W. Stoll, Normal families of non-negative divisors, Math. Z. 84 (1964), 154-218. MR 0165142.

[16] - Value Distribution Theory for Meromorphic Maps, Aspects Math. E7, Friedr. Vieweg, Braunschweig, 1985. MR 0823236. DOI 10.1007/978-3-663-05292-0.

[17] D. D. Thai and S. D. Quang, Uniqueness problem with truncated multiplicities of meromorphic mappings in several complex variables for moving targets, Internat. J. Math. 16 (2005), 903-939. MR 2168074. DOI 10.1142/S0129167X05003132.

[18] — Second main theorem with truncated counting function in several complex variables for moving targets, Forum Math. 20 (2008), 163-179. MR 2386785. DOI 10.1515/FORUM.2008.007. 
[19] D. D. Thai, P. N. T. Trang, and P. D. Huong, Families of normal maps in several complex variables and hyperbolicity of complex spaces, Complex Var. Theory Appl. 48 (2003), 469-482. MR 1979525. DOI 10.1080/0278107031000094963.

[20] Z. Tu, Normality criterions for families of holomorphic mappings of several complex variables into $P^{N}(C)$, Proc. Amer. Math. Soc. 127 (1999), 1039-1049. MR 1469438. DOI 10.1090/S0002-9939-99-04610-9.

[21] - On meromorphically normal families of meromorphic mappings of several complex variables into $P^{N}(C)$, J. Math. Anal. Appl. 267 (2002), 1-19. MR 1886812. DOI 10.1006/jmaa.2000.6770.

[22] Z. Tu and P. Li, Normal families of meromorphic mappings of several complex variables into $P^{N}(C)$ for moving targets, Sci. China Ser. A 48 (2005), suppl., 355-364. MR 2156516. DOI 10.1007/BF02884720.

[23] L. Zalcman, Normal families: New perspectives, Bull. Amer. Math. Soc. (N.S.) 35 (1998), 215-230. MR 1624862. DOI 10.1090/S0273-0979-98-00755-1.

Gerd Dethloff

Université Européenne de Bretagne

France

Université de Brest

Laboratoire de Mathématiques Bretagne Atlantique - UMR CNRS 6205

29238 Brest Cedex 3

France

gerd.dethloff@univ-brest.fr

Do Duc Thai

Department of Mathematics

Hanoi National University of Education

Hanoi

Vietnam

ducthai.do@gmail.com

Pham Nguyen Thu Trang

Department of Mathematics

Hanoi National University of Education

Hanoi

Vietnam

pnttrangsp@yahoo.com 